\title{
The modulational regime of three-dimensional water waves and the Davey-Stewartson system
}

\author{
by \\ Walter CRAIG \\ Department of Mathematics and Lefschetz Center for Dynamical Systems, \\ Brown University, Providence, R.I. 02912, USA \\ E-mail: craig@math.brown.edu \\ and \\ Ulrich SCHANZ and Catherine SULEM \\ Department of Mathematics, University of Toronto, Toronto, M5S $3 \mathrm{G} 3$ Canada. \\ E-mail: schanz@math.toronto.edu; sulem@math.toronto.edu
}

ABSTRACT. - Nonlinear modulation of gravity-capillary waves travelling principally in one direction at the surface of a three-dimensional fluid leads to the Davey-Stewartson system for the wave amplitude and the induced mean flow. In this paper, we present a rigorous derivation of the system and show that the resulting wavepacket satisfies the water wave equations at leading order with precise bounds for the remainder.

Key steps in the analysis are the analyticity of the Dirichlet-Neumann operator with respect to the surface elevation that defines the fluid domain, precise bounds for the Taylor remainders and the description of individual terms in the Taylor series as pseudo-differential operators and their estimates under multiple scale expansions.

Key words: Modulation, water waves.

RÉSUMÉ. - La modulation nonlinéaire d'ondes se propageant principalement dans une direction à la surface d'un canal tridimensionnel conduit au système de Davey-Stewartson pour l'amplitude de l'onde et le champ

A.M.S. classification scheme numbers: 76 B 15, 35 C 10.

Annales de I'Institut Henri Poincaré - Analyse non linéaire - 0294-1449

Vol. 14/97/05/\$ 7.00/

C 1997 L'Association Publications de l'Institut Henri Poincaré. Published by Elsevier B.V. All rights reserved 
moyen induit. Dans cet article, nous présentons une dérivation rigoureuse du système et nous montrons que l'approximation modulationnelle satisfait les équations des ondes de surface à l'ordre dominant.

Les étapes importantes dans l'analyse sont l'analyticité de l'opérateur de Dirichlet-Neumann par rapport à l'interface qui définit le domaine du fluide, des estimations du reste dans le développement de Taylor de l'opérateur, ainsi que la description des différents termes de la série comme opérateurs pseudo-differentiels et leurs développements multi-échelles.

\section{INTRODUCTION}

This paper is a contribution to the mathematical theory of the water wave problem, and the methods of modulational analysis. Our goal is a rigorous understanding of the Davey-Stewartson system as an approximation to the three-dimensional gravity-capillary wave problem, in the modulational scaling regime. This paper extends the previous work of W. Craig, C. Sulem and P.L. Sulem [8] on the two-dimensional water wave in the modulational regime, where the asymptotic description of solutions is given by the cubic Schrödinger equation. The main mathematical contribution for the three (or higher) dimensional problem is the analysis the boundary integral operators of potential theory, which is more intricate than in two dimensions. The description of singular operators and pseudo-differential operators under multiple scale expansions is similar to the analysis of [8].

The water wave problem describes the evolution of an Euler fluid that is inviscid, incompressible and additionally irrotational, with a free surface and under the influence of the gravity and of surface tension. This is a potential flow, which is described in Eulerian coordinates by the velocity field $\mathbf{u}=\nabla \varphi$, where

$$
\Delta \varphi=0
$$

for $\mathbf{x}=\left(x_{1}, x_{2}, x_{3}\right) \in\left\{-h<x_{3}<\eta\left(x_{1}, x_{2}\right),\left(x_{1}, x_{2}\right)=x^{\prime} \in \mathbf{R}^{2}\right\}$ the fluid domain. The bottom boundary condition is that $\partial_{x_{3}} \varphi\left(x^{\prime},-h\right)=0$, and the ctassical free surface conditions are that

$$
\begin{aligned}
& \partial_{t} \varphi+\frac{1}{2}(\nabla \varphi)^{2}+g \eta-\beta H(\eta)=0 \\
& \partial_{t} \eta+\partial_{x^{\prime}} \varphi \cdot \partial_{x^{\prime}} \eta-\partial_{x_{3}} \varphi=0
\end{aligned}
$$


on the surface $x_{3}=\eta\left(x^{\prime}\right)$, where $H(\eta)$ is the mean curvature of the free surface.

In the modulational regime, one derives that solutions to (1.1)-(1.2) are described formally to lowest order in $\epsilon<<1$ by the expressions:

$$
\begin{aligned}
& \widetilde{\eta}=2 \epsilon \operatorname{Re}\left(\frac{i \omega}{g-\beta k_{1}^{2}} c\left(z_{1}, z_{2}, \tau\right) e^{i\left(k_{1} x_{1}-\omega t\right)}\right)+O\left(\epsilon^{2}\right) \\
& \widetilde{\xi}=2 \epsilon \operatorname{Re}\left(c\left(z_{1}, z_{2}, \tau\right) e^{i\left(k_{1} x_{1}-\omega t\right)}\right)+\epsilon d\left(z_{1}, z_{2}, \tau\right)+O\left(\epsilon^{2}\right) .
\end{aligned}
$$

with $z_{1}=\epsilon\left(x_{1}-\omega^{\prime} t\right), z_{2}=\epsilon x_{2}, \tau=\epsilon^{2} t, \omega^{2}=\left(g+\beta k_{1}^{2}\right) k_{1} \tanh \left(h k_{1}\right)$ and $\omega^{\prime}=d \omega / d k_{1}$. The potential function $\tilde{\varphi}(\mathbf{x}, t)$ is the harmonic extension of the boundary values $\tilde{\xi}\left(x^{\prime}, t\right)$ into the fluid domain defined by the upper boundary $\widetilde{\eta}$. The two functions $(c(z, \tau), d(z, \tau))$ satisfy the DaveyStewartson system:

$$
\begin{aligned}
2 i c_{T}+\lambda c_{z_{1} z_{1}}+\mu c_{z_{2} z_{2}} & =\chi|c|^{2} c+\chi_{1} c d_{z_{1}} \\
\alpha d_{z_{1} z_{1}}+d_{z_{2} z_{2}} & =-\gamma|c|_{z_{1}}^{2}
\end{aligned}
$$

with constants $\lambda, \mu, \chi, \chi_{1}, \alpha, \gamma$ that depend upon $g, k, h$ and $\beta$ as specified below.

A fundamental question is in which precise sense does the solution prescribed by the modulational approximation (1.3)-(1.4) approximate the full Euler equations (1.1)-(1.2) which give the fluid evolution. In this paper, we give a rigorous derivation of the Davey-Stewartson system (1.4), together with an estimate of the error of the approximation (1.3), which is in the same spirit as the paper [8] on the two-dimensional water wave problem. The modulational regime is derived with the method of multiple scales (both spatial and temporal), using the basic assumption that the solutions behave independently on asymptotic separated scales. In general, the method of multiple scales involves singular pertubations, as the description of slow time and/or spatial scales ultimately results in the replacement of higher derivative operators with lower. Justifying this formal analysis gives rise to a number of basic mathematical questions, as in general the critical analytic issues involve the highest order differential operators and the behavior of solutions at high wavenumbers. A further consideration for the water wave problem is that the integral operators of potential theory for the fluid domain play a central role: under multiple scales analysis these are approximated by differential operators, and the nature of this approximation must be understood. A general theory of pseudo-differential operators and multiple scale expansions is developed for this purpose in [8], and this with several modifications will be used in this paper. 
The original derivations of the modulation equations for threedimensional water waves appeared in Benney and Roskes [2] and in Davey-Stewartson [10] in the case of pure gravity waves. The effect of surface tension was analysed by Djordjevic and Redekopp [11] as well as in Ablowitz and Segur [1]. The derivation of the nonlinear Schrödinger equation from the two-dimensional water wave problem was first obtained by Zakharov [27] in the case of infinitely deep water, and in finite depth water by Hasimoto and Ono [15]. As opposed to our analysis in [8] in which Lagrangian variables were used, we adopt in the present paper an Eulerian approach, using dependent variables defined on the free surface alone which are described in Craig and Sulem [9]. Formal aspects of the modulational analysis are thus quite similar to that of Hasimoto and Ono [15] for the two dimensional problem. This choice of coordinates is not necessarily optimal for the initial value problem for water waves, but they do allow a relatively clean and systematic treatment of the multiple scales analysis and an estimate of remainder terms.

Asymptotic approximations of the water wave problem have been the origin of many of the nonlinear partial differential equations of mathematical physics [22]. Because of this, there has been an effort over a number of years to understand in a rigorous way the validity of these approximations and to justify if possible the use of the asymptotic limits. Kano and Nishida [17] proved an existence theorem for the initial value problem for two dimensional water waves for analytic initial data and gave a rigorous analysis of the shallow water scaling limit. In subsequent papers, both Craig [6] and Kano and Nishida [18] addressed the dispersive long wave scaling regimes for two-dimensional water waves, giving an analysis of the Boussinesq and KdV approximations. The modulational scaling regimes are somehow harder to study, as the solution is approximated through an ansatz of a multiple scale analysis, which involves in particular the assumption of the independence of several scaling regimes, and this independence must be justified with rigorous errors estimates in the full Euler equations (1.1)(1.2). For the two-dimensional problem, a rigorous result on the derivation of the nonlinear Schrödinger equation is given in [8]. The results in the three-dimensional modulational regime of this paper are close in spirit to $[8]$.

There are a number of recent papers on rigorous justification of modulational analysis in several settings other than the water wave problem. The work of Collet and Eckmann [5] gives a comparison of solutions of the Swift-Hohenberg equation with a modulational approximation in the form of a Ginzburg-Landau equation. Both of these are parabolic equations. Mielke 
and Schneider have also studied parabolic problems whose modulational limit gives the Ginzburg-Landau equation, and additionally to these, Kirrmann, Mielke and Schneider [19] have results for modulational regimes of nonlinear hyperbolic systems. More recently, Pierce and Wayne [25] studied the modulational regime for a one-dimensional wave equation which involves the interaction of left and right moving periodic wave trains. The modulational regime and the method of multiple scales are widespread in applied mathematics, and we think that a rigorous mathematical study of this approximation procedure is important to understand the nature of the asymptotic solution.

The organization of the paper is as follows. In Section 2, we describe the water wave problem in terms of the variables on the free surface $\left(\eta\left(x^{\prime}, t\right), \xi\left(x^{\prime}, t\right)=\varphi\left(x^{\prime}, \eta\left(x^{\prime}, t\right), t\right)\right)$, the Dirichlet-Neumann operator $G(\eta)$ for the fluid domain and its formal Taylor series expansion with respect to $\eta\left(x^{\prime}\right)$. Section 3 is devoted to the formal derivation of the Davey-Stewartson system. 'The analysis starts in Section 4, where we give the rigorous analysis of the Dirichlet-Neumann operator and its Taylor approximation. In concise terms, we show that, for surface variations $\eta\left(x^{\prime}\right)$ in a neighborhood of zero in the $C^{1}\left(\mathbf{R}^{2}\right)$-topology such that $\left|\partial_{x}^{s+1} \eta\right|_{L^{\infty}}<\infty$, the operator $G(\eta)$ is analytic as a mapping between the Sobolev spaces $G(\eta): W^{s+1, q}\left(\mathbf{R}^{2}\right) \rightarrow W^{s, q}\left(\mathbf{R}^{2}\right)$ for any $1<q<\infty$. This work on the regularity of operators of potential theory under pertubation of the domain is related to early work of Garabedian and Schiffer [12], and to work of Coifman and Meyer [4] on Cauchy integrals. Our analysis of the analyticity of $G(\eta)$ is fundamentally based on the multiple commutator estimates that appear in the paper of Christ and Journé [3]. Section 5 gives an estimate in $W^{s, q}\left(\mathbf{R}^{2}\right)$ of a class of singular integral operators that make up the Dirichlet-Neumann operator, its Taylor approximates and its Taylor remainders. Section 6 is focussed on the mathematical justification of the formal expansion for the water wave problem given in Section 3. In part, this uses an analysis of pseudo-differential operators in a multiple scale regime, which is along the lines of [8]. As we work here in general $L^{q}\left(\mathbf{R}^{2}\right)$-spaces, the required additional estimates are provided.

We now conclude this introduction by discussing the form of justification of the modulational limit that we can provide. In both the two-dimensional and three-dimensional water wave problem, the modulational approximation involves solutions in the form of wavepackets of amplitude $O(\epsilon)$, whose envelopes simply translate with the group velocity over time intervals of length $O\left(\epsilon^{-1}\right)$, and evolve on time intervals of length $O\left(\epsilon^{-2}\right)$ according to a modulational equation (nonlinear Schrödinger or Davey-Stewartson 
equations). In order to compare a solution to the water wave problem to its modulational approximation, it is thus necessary to have an existence theorem for solutions of (1.1)-(1.2) for initial data of amplitude $O(\epsilon)$ which have time intervals of existence of $O\left(\epsilon^{-2}\right)$, corresponding to times $O(1)$ in the modulated time scale. At present, there are no existence theorems for the full water wave problem, so that we are as yet unable to make the statement that true solutions $(\eta, \xi)$ of $(1.1)-(1.2)$ and their modulational approximations $(\widetilde{\eta}, \widetilde{\xi})$ of $(1.3)-(1.4)$ remain close in an appropriate norm over modulational time scales: $\sup _{0 \leq t \leq O\left(\epsilon^{-2}\right)}\|(\eta, \xi)-(\widetilde{\eta}, \widetilde{\xi})\|<o\left(\epsilon^{3}\right)$. In this paper, we make the alternative, and weaker statement that the approximations $(\widetilde{\eta}, \widetilde{\xi})$ constructed from the Davey-Stewartson system (1.4) result in an $o\left(\epsilon^{3}\right)$ error when acted upon by the nonlinear water wave operator given by the r.h.s of (1.2). If the implicit function theorem were available, these two statements would be equivalent but as it is not, the second is weaker than the first. The statement that we make is sufficient to guarantee that over time scales of interest $\left(0 \leq l<O\left(\epsilon^{-2}\right)\right)$, the dominant evolution of the modulational regime is described through (1.3) and the Davey-Stewartson system (1.4), and accumulated errors cannot grow to significance.

The construction of approximate solutions in the modulational regime also depends upon the well-posedness of the initial value problem for the Davey-Stewartson system (1.4). There are two choices of sign for each of the quantities $\alpha$ and $\lambda / \mu$ which affect this (although $\alpha<0$, $\lambda / \mu<0$ does not occur in the water wave problem). All of these cases have been addressed in the literature, see Ghidalia and Saut [13], Linares and Ponce [20], Guzmán-Gómez [14] and Hayashi and Saut [16]. The nature of the solution depends importantly upon the sign of $\alpha$. For $\alpha>0$, one can take $c_{0}(z) \in H^{m}\left(\mathbf{R}^{2}\right)$ and essentially one has solutions $(c(z, \tau), d(z, \tau)) \in H^{m}\left(\mathbf{R}^{2}\right) \times H^{m+1}\left(\mathbf{R}^{2}\right)$. When however $\alpha<0$, one cannot impose zero boundary conditions for $d(z, \tau)$ at spatial infinity. The solution essentially has a "wake" of infinite extent backwards along the characteristics $z_{1} \pm \sqrt{-\alpha} z_{2}=$ Const, and $d(z, \tau) \in W^{m-1 . \infty}\left(\mathbf{R}^{2}\right)$ and not better. A local representation of the solutions of the nonlocal equations (1.2) is then problematic. Our results on the justification of the modulational approximation reflect this fact: we have results in the case $\alpha<0$ only for the approximate solutions of (1.3) when cutoff smoothly near (arbitrarily close to) the modulational variables $z$ at spatial infinity. These results on the initial value problem are reviewed in Section 6, which then finishes with the proof of our main results and rigorous error estimates. 


\section{EQUATIONS OF MOTION}

\subsection{The water wave problem}

We consider the movement of the free surface $\mathbf{x}=\left(x^{\prime}, \eta\left(x^{\prime}\right)\right)$, $x^{\prime}=\left(x_{1}, x_{2}\right) \in \mathbf{R}^{2}$, of a three-dimensional fluid with surface tension $\beta$, under the influence of gravity $g$. The domain is a channel which is infinite in the horizontal directions and has a fixed bottom at $x_{3}=-h$. The fluid is taken to be incompressible, inviscid and irrotational, so that the fluid motion is described by a velocity potential $\varphi$ which satisfies:

$$
\Delta \varphi=0 \quad \text { for } \quad-h<x_{3}<\eta\left(x^{\prime}, t\right) .
$$

The boundary conditions are

$$
\partial_{x_{3}} \varphi=0 \quad \text { on } \quad x_{3}=-h
$$

and on $x_{3}=\eta\left(x^{\prime}, t\right)$, which is the free surface over the fluid domain,

$$
\begin{aligned}
& \partial_{t} \varphi+\frac{1}{2}(\nabla \varphi)^{2}+g \eta-\beta H(\eta)=0, \\
& \partial_{t} \eta+\partial_{x^{\prime}} \varphi \cdot \partial_{x^{\prime}} \eta-\partial_{x_{3}} \varphi=0
\end{aligned}
$$

where

$$
H(\eta)=\frac{\partial_{x_{1}}^{2} \eta\left(1+\left(\partial_{x_{2}} \eta\right)^{2}\right)+\partial_{x_{2}}^{2} \eta\left(1+\left(\partial_{x_{1}} \eta\right)^{2}\right)-2 \partial_{x_{1}} \eta \partial_{x_{2}} \eta \partial_{x_{1}} \partial_{x_{2}} \eta}{\left(1+\left|\partial_{x^{\prime}} \eta\right|^{2}\right)^{3 / 2}}
$$

is the mean curvature of the free surface. The aim of this section is to reduce the system (2.1)-(2.3) to a system where all the functions are evaluated at the free surface only and $\varphi$ and its derivatives in the interior are not used. For this purpose, we introduce the trace of the velocity potential $\varphi$ at the surface

$$
\xi\left(x^{\prime}, t\right)=\varphi\left(x^{\prime}, \eta\left(x^{\prime}, t\right), t\right)
$$

and the Dirichlet-Neumann operator acting on $\xi\left(x^{\prime}\right)$, which is defined by

$$
G(\eta) \xi=\sqrt{1+\left|\partial_{x^{\prime}} \eta\right|^{2}} \partial_{n} \varphi=\left.\left(-\partial_{x^{\prime}} \varphi \cdot \partial_{x^{\prime}} \eta+\partial_{x_{3}} \varphi\right)\right|_{x_{3}=\eta\left(x^{\prime}, t\right)},
$$

where $\partial_{n} \varphi$ is the normal derivative of $\varphi$ on the surface. The linear operator $G(\eta)$ relates (up to a normalization factor) the boundary values of $\varphi$ on Vol. 14, n 5-1997. 
the surface to its normal derivative. On the free surface $x_{3}=\eta\left(x^{\prime}, t\right)$ we additionally have that

$$
\partial_{t} \varphi=\partial_{t} \xi-\partial_{t} \eta \partial_{x_{3}} \varphi ; \quad \partial_{x_{i}} \varphi=\partial_{x_{i}} \xi-\partial_{x_{i}} \eta \partial_{x_{3}} \varphi \quad(i=1,2)
$$

and

$$
\partial_{x_{3}} \varphi=\frac{G(\eta) \xi+\partial_{x^{\prime}} \eta \cdot \partial_{x^{\prime}} \xi}{1+\left|\partial_{x^{\prime}} \eta\right|^{2}}
$$

Using expressions (2.4)-(2.6), equations (2.1)-(2.3) are equivalent to the system

$$
\begin{aligned}
& \partial_{t} \eta-G(\eta) \xi=0 \\
& \partial_{t} \xi+g \eta+\frac{1}{2\left(1+\left|\partial_{x^{\prime}} \eta\right|^{2}\right)} \\
& \quad \times\left(\left|\partial_{x^{\prime}} \xi\right|^{2}-(G(\eta) \xi)^{2}-2\left(\partial_{x^{\prime}} \eta \cdot \partial_{x^{\prime}} \xi\right) G(\eta) \xi\right. \\
& \left.\quad+\left|\partial_{x^{\prime}} \eta\right|^{2}\left|\partial_{x^{\prime}} \xi\right|^{2}-\left(\partial_{x^{\prime}} \eta \cdot \partial_{x^{\prime}} \xi\right)^{2}\right)-\beta H(\eta)=0
\end{aligned}
$$

which is an evolution equation for the elevation of the free surface $\eta\left(x^{\prime}, t\right)$ and the trace of the velocity potential on the free surface $\xi\left(x^{\prime}, t\right)$. It is this system that we will use in this paper on the rigorous modulational analysis of the three-dimensional water wave problem.

\subsection{The Taylor expansion of $G$}

We briefly outline the formal derivation of the Taylor expansion of $G$ in powers of the surface elevation $\eta$. Details can be found in [8] for the two-dimensional case and in [7] for the three-dimensional case. We look for an expansion of the form

$$
G(\eta)=\sum_{j=0}^{\infty} G_{j}(\eta)
$$

where $G_{j}(\eta)$ is a pseudo-differential operator homogeneous in $\eta$ of degree $j$. For this, we consider the particular family of harmonic functions:

$$
\varphi_{p}\left(x^{\prime}, x_{3}\right)=e^{i p \cdot x^{\prime}} \cosh \left(|p|\left(x_{3}+h\right)\right)
$$

where $p=\left(p_{1}, p_{2}\right) \in \mathbf{R}^{2}$ and $|p|=\sqrt{p_{1}^{2}+p_{2}^{2}}$. These are harmonic functions in $\left\{x_{3}>-h\right\}$ which satisfy $\partial_{x_{3}} \varphi_{p}=0$ on the bottom boundary $x_{3}=-h$. By definition,

$$
G(\eta) \varphi_{p}=\partial_{x_{3}} \varphi_{p}-\partial_{x^{\prime}} \varphi_{p} \cdot \partial_{x^{\prime}} \eta_{x^{\prime}}
$$


We substitute (2.9) into (2.10), expand the hyperbolic functions near $x_{3}=0$, and replace the r.h.s. of (2.10) by its expansion $\sum_{j=0}^{\infty} G_{j}(\eta) \varphi_{p}$. We obtain thus an identity, and by identifying the terms of degree $j$ in $\eta$ we get the expansion of $G$ from a recursion formula. Using the usual notation that $D_{\ell}=-i \partial_{x_{\ell}}$ and $|D|=(-\Delta)^{1 / 2}$, the result is as follows. For $j$ even:

$$
\begin{aligned}
G_{j}(\eta)= & \frac{1}{j !}\left(\eta^{j}|D|^{j+1} \tanh (h|D|)-i \partial_{x^{\prime}}\left(\eta^{j}\right) \cdot D|D|^{j-1} \tanh (h|D|)\right) \\
& -\sum_{\substack{\ell<j \\
\ell \text { even }}} \frac{1}{(j-\ell) !} G_{\ell}(\eta) \eta^{j-\ell}|D|^{j-\ell} \\
& -\sum_{\substack{\ell<j \\
\ell \text { odd }}} \frac{1}{(j-\ell) !} G_{\ell}(\eta) \eta^{j-\ell}|D|^{j-\ell} \tanh (h|D|)
\end{aligned}
$$

For $j$ odd:

$$
\begin{aligned}
G_{i}(\eta) & =\frac{1}{j !}\left(\eta^{j}|D|^{j+1}-i \partial_{x^{\prime}}\left(\eta^{j}\right) \cdot D|D|^{j-1}\right) \\
& -\sum_{\substack{\ell<j \\
\ell \text { even }}} \frac{1}{(j-\ell) !} G_{\ell}(\eta) \eta^{j-\ell}|D|^{j-\ell} \tanh (h|D|) \\
& -\sum_{\substack{\ell<j \\
\ell \text { odd }}} \frac{1}{(j-\ell) !} G_{\ell}(\eta) \eta^{j-\ell}|D|^{j-\ell}
\end{aligned}
$$

In the analysis of this paper, we need the explicit form of the first three terms of the expansion, namely

$$
\begin{aligned}
& G_{0}=|D| \tanh (h|D|) \\
& G_{1}(\eta)=D \cdot \eta D-G_{0} \eta G_{0} \\
& G_{2}(\eta)=-\frac{1}{2}\left(G_{0} \eta^{2}|D|^{2}+|D|^{2} \eta^{2} G_{0}-2 G_{0} \eta G_{0} \eta G_{0}\right) .
\end{aligned}
$$

This form of analysis of the Dirichlet-Neumann operator is useful in a variety of settings. For example, we have used it in a method for numerical computations of time dependent free surface flows [9], and de la Llave and Panayotaros [21] in a similar context have derived the Taylor expansion of the Dirichlet-Neumann operator in the context of water waves on the surface of a fluid layer surrounding a gravitating sphere. 


\section{FORMAL DERIVATION OF MODULATED SOLUTIONS}

This section is devoted to the formal modulation expansion, leading to the Davey-Stewartson system. This derivation starts with the form (2.7)-(2.8) of the water wave problem, but in other respects follows the general method of multiple scales. The modulational regime considers small amplitude solutions of (2.7)-(2.8); the linearized system around water at rest is

$$
L\left(\begin{array}{l}
\eta \\
\xi
\end{array}\right)=0
$$

with

$$
L=\left(\begin{array}{cc}
\partial_{t} & -G_{0} \\
g-\beta \Delta & \partial_{l}
\end{array}\right) .
$$

The system (3.1) admits solutions of the form:

$$
\eta=\frac{i \omega}{g+\beta|k|^{2}} c e^{i \varphi}+c . c, \quad \xi=c e^{i \varphi}+c . c+d
$$

where $\varphi=k \cdot x^{\prime}-\omega t$. We use $c . c$ to denote the complex conjugate of the preceding terms. The constants $c$ and $d$ are arbitrary, while the wave vector $k=\left(k_{1}, k_{2}\right)$ is related to $\omega$ by the dispersion relation;

$$
\omega^{2}(k)=\left(g+\beta|k|^{2}\right)|k| \tanh (h|k|)
$$

We study a scaling regime suitable to observe a packet of nearly onedimensional waves $\left(\left|k_{2}\right| \ll\left|k_{1}\right|\right)$ travelling in the $x_{1}$-direction. We suppose that the waves have small amplitudes and that the effect of the nonlinearity will be to modulate the amplitude $c$ which becomes a slowly varying function of space and time. To this end, we introduce a multiple scale expansion, with a large scale spatial variable $X^{\prime}=\left(X_{1}, X_{2}\right)=\epsilon x^{\prime}=$ $\epsilon\left(x_{1}, x_{2}\right)$ and two slow times $T=\epsilon t$ and $\tau=\epsilon^{2} t$, and we expand the solution in the form

$$
\begin{aligned}
& \eta=\epsilon \eta^{(1)}+\epsilon^{2} \eta^{(2)}+\cdots \\
& \xi=\epsilon \xi^{(1)}+\epsilon^{2} \xi^{(2)}+\cdots
\end{aligned}
$$

and

$$
G=G^{(0)}+\epsilon G^{(1)}+\cdots
$$


At leading order, we have

$$
\begin{aligned}
\eta^{(1)} & =\frac{i \omega}{g+\beta k_{1}^{2}} c(X, T, \tau) e^{i \varphi}+c . c \\
\xi^{(1)} & =c(X, T, \tau) e^{i \varphi}+c . c+d(X, T, \tau) .
\end{aligned}
$$

From the preceding paragraph,

$$
\varphi=k_{1} x_{1}-\omega t \quad \text { and } \quad \omega^{2}=\left(g+\beta k_{1}^{2}\right) k_{1} \tanh \left(h k_{1}\right) .
$$

For concise notation we will use the notations in the following calculations: $\chi=h k_{1}, \sigma=\tanh \chi$. Furthermore, $D_{j}=D_{j}^{(0)}+\epsilon D_{j}^{(1)}$ where derivatives are $D_{j}^{(0)}=\frac{1}{i} \partial_{x_{j}}$ and $D_{j}^{(1)}=\frac{1}{i} \partial_{X_{j}}(j=1,2)$. We first write the expression of $|D|$ and $\tanh (h|D|)$ using results of [8] on pseudo-differential operators in a multiple scale regime, obtaining

$$
\begin{aligned}
|D|=\left|D_{1}^{(0)}\right|+\epsilon & D_{1}^{(0)}\left|D_{1}^{(0)}\right|^{-1} D_{1}^{(1)}+\frac{\epsilon^{2}}{2}\left|D_{1}^{(0)}\right|^{-1} D_{2}^{(1) 2}+O\left(\epsilon^{3}\right) \\
\tanh (h|D|)= & \tanh \left(h\left|D_{1}^{(0)}\right|\right) \\
& +\epsilon h D_{1}^{(0)}\left|D_{1}^{(0)}\right|^{-1}\left(1-\tanh ^{2}\left(D_{1}^{(0)}\right)\right) D_{1}^{(1)} \\
& +\epsilon^{2}\left(\frac{h}{2}\left|D_{1}^{(0)}\right|^{-1}\left(1-\tanh ^{2}\left(h D_{1}^{(0)}\right)\right) D_{2}^{(1) 2}\right. \\
& \left.-h^{2}\left(1-\tanh ^{2}\left(h D_{1}^{(0)}\right)\right) \tanh \left(h\left|D_{1}^{(0)}\right|\right)\right) D_{1}^{(1) 2} \\
& +O\left(\epsilon^{3}\right)
\end{aligned}
$$

To obtain the coefficients $G^{(n)}$, we first write the expansion of $G$ in terms of powers of $\eta$ (see section 2.2) and then use the expansion of $\eta$ in $\epsilon$ together with (3.7)-(3.8). At leading order, we recover

$$
G^{(0)}=D_{1}^{(0)} \tanh \left(h D_{1}^{(0)}\right)
$$

The terms of order $\epsilon$ and $\epsilon^{2}$ are respectively

$$
\begin{aligned}
G^{(1)}= & D_{1}^{(0)} \eta^{(1)} D_{1}^{(0)}-G^{(0)} \eta^{(1)} G^{(0)}+\tanh \left(h D_{1}^{(0)}\right) D_{1}^{(1)} \\
& +h D_{1}^{(0)}\left(1-\tanh ^{2}\left(h D_{1}^{(0)}\right)\right) D_{1}^{(1)}
\end{aligned}
$$


and

$$
\begin{aligned}
G^{(2)}= & \frac{1}{2}\left|D_{1}^{(0)}\right|^{-1} \tanh \left(h\left|D_{1}^{(0)}\right|\right) D_{2}^{(1) 2} \\
& +h\left(1-\tanh ^{2}\left(h D_{1}^{(0)}\right)\right) D_{1}^{(1) 2}+\frac{h}{2}\left(1-\tanh ^{2}\left(h D_{1}^{(0)}\right)\right) D_{2}^{(1) 2} \\
& -h^{2} D_{1}^{(0)}\left(1-\operatorname{anh}^{2}\left(h D_{1}^{(0)}\right)\right) \tanh \left(h D_{1}^{(0)}\right) D_{1}^{(1) 2} \\
& +D_{1}^{(0)} \eta^{(2)} D_{1}^{(0)}-G^{(0)} \eta^{(2)} G^{(0)}+D_{1}^{(0)} \eta^{(1)} D_{1}^{(1)}+D_{1}^{(1)} \eta^{(1)} D_{1}^{(0)} \\
& -G^{(0)} \eta^{(1)}\left(\tanh \left(h D_{1}^{(0)}\right) D_{1}^{(1)}+h D_{1}^{(0)}\left(1-\tanh ^{2}\left(h D_{1}^{(0)}\right)\right) D_{1}^{(1)}\right) \\
& -\left(\tanh \left(h D_{1}^{(0)}\right) D_{1}^{(1)}+h D_{1}^{(0)}\left(1-\tanh ^{2}\left(h D_{1}^{(0)}\right)\right) D_{1}^{(1)}\right) \eta^{(1)} G^{(0)} \\
& -\frac{1}{2}\left(G^{(0)} \eta^{(1) 2} D_{1}^{(0) 2}+D_{1}^{(0) 2} \eta^{(1) 2} G^{(0)}-2 G^{(0)} \eta^{(1)} G^{(0)} \eta^{(1)} G^{(0)}\right)
\end{aligned}
$$

We now expand equations (2.7)-(2.8) in powers of $\epsilon$, which at order $n$ for $n>1$ gives the inhomogeneous linear system;

$$
L\left(\begin{array}{l}
\eta^{(n)} \\
\xi^{(n)}
\end{array}\right)=\left(\begin{array}{l}
A_{n} \\
B_{n}
\end{array}\right) .
$$

The solvability condition requires that the r.h.s. of (3.12) is orthogonal to the kernel of the adjoint operator

$$
L^{*}=\left(\begin{array}{cc}
-\partial_{t} & g+\beta D_{1}^{(0) 2} \\
-G^{(0)} & -\partial_{t}
\end{array}\right) .
$$

The kernel of $L^{*}$ is spanned by

$$
\left(\begin{array}{c}
1 \\
\frac{-i \omega}{g+3 k_{1}^{2}}
\end{array}\right) e^{i \varphi} \text { and }\left(\begin{array}{l}
1 \\
0
\end{array}\right),
$$

thus the solvability conditions are equivalent to the following two conditions:

(S1) $A_{n}$ does not contain terms independent of $\varphi$

(S2) The coefficients $P_{n}$ and $Q_{n}$ of $e^{i \varphi}$ in $A_{n}$ and $B_{n}$ respectively satisfy

$$
P_{n}+\frac{i \omega}{g+\beta k_{1}^{2}} Q_{n}=0 \text {. }
$$

At order 2, we have

$$
\begin{aligned}
& A_{2}=-\eta_{T}^{(1)}+G^{(1)} \xi^{(1)} \\
& B_{2}=-\xi_{T}^{(1)}-\frac{1}{2}\left(\xi_{x_{1}}^{(1)}\right)^{2}+\frac{1}{2}\left(G^{(0)} \xi^{(1)}\right)^{2}+2 \beta \eta_{x_{1} X_{1}}^{(1)} .
\end{aligned}
$$


Using the expressions of $\eta^{(1)}, \xi^{(1)}$ and $G^{(1)}$ given in (3.6) and (3.10) we get:

$$
\begin{aligned}
A_{2}= & i\left(\frac{-\omega}{g+\beta k_{1}^{2}} c_{T}-\left(\sigma+\chi\left(1-\sigma^{2}\right)\right) c_{X_{1}}\right) e^{i \varphi} \\
& +\frac{2 i \omega k_{1}^{2}}{g+\beta k_{1}^{2}} c^{2}(1-\sigma \tanh (2 \chi)) e^{2 i \varphi}+c . c \\
B_{2}= & -d_{T}+k_{1}^{2}\left(\sigma^{2}-1\right)|c|^{2}-\left(c_{T}+\frac{2 \omega k_{1} \beta}{g+\beta k_{1}^{2}} c_{X_{1}}\right) e^{i \varphi} \\
& +\frac{1}{2} k_{1}^{2} c^{2}\left(1+\sigma^{2}\right) e^{2 i \varphi}+c . c
\end{aligned}
$$

At this order, the solvability condition is that

$$
\frac{2 \omega}{g+\beta k_{1}^{2}} c_{T}+\left(\sigma+\chi\left(1-\sigma^{2}\right)+\frac{2 \beta k_{1}^{2} \sigma}{g+\beta k_{1}^{2}}\right) c_{\mathrm{x}_{1}}=0
$$

or equivalently,

$$
c_{T}+\omega^{\prime} c_{X_{1}}=0
$$

where $\omega^{\prime}=\partial_{k_{1}} \omega\left(k_{1}, 0\right)$. Thus $c=c\left(z_{1}, z_{2}, \tau\right)$ with $z_{1}=X_{1}-\omega^{\prime} T$, and $z_{2}=X_{2}$. This expresses that the wave packet travels with its linear group velocity $\omega^{\prime}$. The system (3.12) at order 2 is solved in the form

$$
\begin{aligned}
& \eta^{(2)}=p_{1} e^{i \varphi}+p_{2} e^{2 i \varphi}+c . c+p_{3} \\
& \xi^{(2)}=q_{1} e^{i \varphi}+q_{2} e^{2 i \varphi}+c . c+q_{3}
\end{aligned}
$$

with

$$
\begin{aligned}
& p_{1}=\frac{1}{g+\beta k_{1}^{2}} c_{T}+\frac{1}{\omega}\left(\sigma+\chi\left(1-\sigma^{2}\right)\right) c_{X_{1}} \\
& p_{2}=\frac{\left(\sigma^{2}-3\right) k_{1}^{2}}{2\left(\beta k_{1}^{2}\left(\sigma^{2}-3\right)+\sigma^{2} g\right)} c^{2} \\
& q_{1}=0 \\
& q_{2}=\frac{i \omega k_{1}\left(2 \frac{g+4 \beta k_{1}^{2}}{g+\beta k_{1}^{2}}\left(1-\sigma^{2}\right)+\frac{1}{2}\left(1+\sigma^{2}\right)^{2}\right)}{4 \sigma\left(\beta k_{1}^{2}\left(\sigma^{2}-3\right)+\sigma^{2} g\right)} c^{2} \\
& p_{3}=\frac{1}{g}\left(k_{1}^{2}\left(\sigma^{2}-1\right)|c|^{2}-d_{x}\right) .
\end{aligned}
$$

The dependent variable $q_{3}$ can be chosen equal to 0 , and $c$ and $d$, are slowly varying functions which are not determined at this stage of Vol. $14, n^{\circ}$ 5-1997. 
the expansion. Note that the denominator of $p_{2}$ and $q_{2}$ vanishes when $\left.\beta k_{1}^{2}\left(\sigma^{2}-3\right)+\sigma^{2} g\right)=0$. This is known as the second harmonic resonance [11]. At wavenumbers satisfying this condition, the analysis breaks down and a new scaling is required. A formal analysis of this regime can be found in the article of McGoldrick [23] and we do not discuss it in the present paper.

For wavenumbers such that $\left.\beta k_{1}^{2}\left(\sigma^{2}-3\right)+\sigma^{2} g\right) \neq 0$, we have at order $\epsilon^{3}$ :

$$
\begin{aligned}
A_{3}= & -\eta_{T}^{(2)}-\eta_{\tau}^{(1)}+G^{(2)} \xi^{(1)}+G^{(1)} \xi^{(2)} \\
B_{3}= & -\xi_{T}^{(2)}-\xi_{\tau}^{(1)}-\xi_{x_{1}}^{(1)} \xi_{X_{1}}^{(1)}-\xi_{x_{1}}^{(1)} \xi_{x_{1}}^{(2)} \\
& +G^{(0)} \xi^{(1)}\left(G^{(0)} \xi^{(2)}+G^{(1)} \xi^{(1)}\right) \\
& +\eta_{x_{1}}^{(1)} \xi_{x_{1}}^{(1)} G^{(0)} \xi^{(1)} \\
& +\beta\left(\eta_{X_{1} X_{1}}^{(1)}+\eta_{X_{2} X_{2}}^{(1)}+2 \eta_{x_{1} X_{1}}^{(2)}-\frac{3}{2} \eta_{x_{1} x_{1}}^{(1)} \eta_{x_{1}}^{(1) 2}\right)
\end{aligned}
$$

The elimination of the $\varphi$-independent term in $A_{3}$ leads to:

$$
-p_{3 T}-h d_{X_{1} X_{1}}-h d_{X_{2} X_{2}}-\frac{2 \omega k_{1}}{g+\beta k_{1}^{2}}|c|_{z_{1}}^{2}=0
$$

or equivalently

$$
-d_{T T}+g h d_{X_{1} X_{1}}+g h d_{X_{2} X_{2}}+\alpha_{1}|c|_{X_{1}}^{2}=0
$$

with

$$
\alpha_{1}=\frac{2 \omega g k_{1}}{g+\beta k_{1}^{2}}+\omega^{\prime}\left(1-\sigma^{2}\right) k_{1}^{2} .
$$

Using (3.18), we see that $U=d_{T}+\omega^{\prime} d_{X_{1}}$ satisfies the homogeneous wave equation

$$
-U_{T T}+g h\left(U_{X_{1} X_{1}}+U_{X_{2} X_{2}}\right)=0
$$

In the limit $T$ large, or equivalently $\tau=O(1)$ (which is the regime that interests us), $U=0$, therefore we might as well assume that $d$, similarly to $c$, is a function of $z_{1}, z_{2}$ and $\tau$ only. Equation (3.22) thus reduces to

$$
\left(g h-\omega^{\prime 2}\right) d_{z_{1} z_{1}}+g h d_{z_{2} z_{2}}+\alpha_{1}|c|_{z_{1}}^{2}=0
$$

For wave numbers such that $\omega^{\prime 2}=g h,(3.23)$ is singular and a different scaling has to be used [11]. In the following, we assume that this coefficient does not vanish. 
To express the solvability condition ( $S 2$ ) we write $P_{3}$ and $Q_{3}$ in the form

$$
\begin{aligned}
P_{3}= & -p_{1 T}-\frac{i \omega}{g+\beta k_{1}^{2}} c_{\tau}-\frac{1}{2 k_{1}}\left(\sigma+\chi\left(1-\sigma^{2}\right)\right) c_{z_{2} z_{2}} \\
& -h\left(1-\sigma^{2}\right)(1-\chi \sigma) c_{z_{1} z_{1}}+k_{1}^{2}\left(1-\sigma^{2}\right) p_{3} c \\
& -\frac{2 i \omega k_{1}^{2}}{g+\beta k_{1}^{2}}(1-\sigma \tanh (2 \chi)) c^{*} q_{2}-k_{1}^{2}\left(1+\sigma^{2}\right) c^{*} p_{2} \\
& +\frac{\omega k_{1}}{g+\beta k_{1}^{2}} c d_{z_{1}}+\frac{k_{1}^{3} \sigma \omega^{2}}{\left(g+\beta k_{1}^{2}\right)^{2}}\left(-1+2 \sigma \tanh (2 \chi)|c|^{2} c\right) \\
Q_{3}= & -c_{\tau}-i k_{1} c d_{z_{1}}-2 k_{1}^{2}(1-\sigma \tanh (2 \chi)) c^{*} q_{2} \\
& +\frac{i \omega \beta}{g+\beta k_{1}^{2}}\left(c_{z_{1} z_{1}}+c_{z_{2} z_{2}}\right)+2 i k_{1} \beta p_{1} z_{1} \\
& +\frac{3}{2} i \beta k_{1}^{4}\left(\frac{\omega}{g+\beta k_{1}^{2}}\right)^{3}|c|^{2} c \\
& +\frac{i k_{1}^{3} \omega \sigma}{g+\beta k_{1}^{2}}(1-2 \sigma \tanh (2 \chi))|c|^{2} c
\end{aligned}
$$

Solvability conditions $(S I)(S 2)$ read

$$
\begin{aligned}
2 i c_{\tau}+\lambda c_{z_{1} z_{1}}+\mu c_{z_{2} z_{2}} & =\chi|c|^{2} c+\chi_{1} c d_{z_{1}} \\
\alpha d_{z_{1} z_{1}}+d_{z_{2} z_{2}} & =-\gamma|c|_{z_{1}}^{2}
\end{aligned}
$$

where, using notation similar to Ablowitz and Segur [1], the constants are given in the following list:

$$
\begin{aligned}
& \lambda=\partial_{k_{1}}^{2} \omega\left(k_{1}, 0\right) \equiv \omega^{\prime \prime} \\
& \mu= \omega^{\prime} / k_{1} \\
& \chi=\frac{k_{1}^{4}}{2 \omega}\left(\frac{\left(1-\sigma^{2}\right)\left(9-\sigma^{2}\right)+\widetilde{\beta}\left(3-\sigma^{2}\right)\left(7-\sigma^{2}\right)}{\sigma^{2}-\widetilde{\beta}\left(3-\sigma^{2}\right)}\right. \\
&\left.\quad+8 \sigma^{2}-2\left(1-\sigma^{2}\right)^{2}(1+\widetilde{\beta})-\frac{3 \sigma^{2} \widetilde{\beta}}{1+\widetilde{\beta}}\right) \\
& \chi_{1}=-k_{1}\left(2+\frac{\omega^{\prime} k_{1}}{\omega}\left(1-\sigma^{2}\right)(1+\widetilde{\beta})\right) \\
& \alpha= \frac{g h-\omega^{\prime 2}}{g h} \\
& \gamma=\frac{k_{1}}{g h}\left(\frac{2 \omega g}{g+\beta k_{1}^{2}}+\omega^{\prime}\left(1-\sigma^{2}\right) k_{1}\right) \\
& \widetilde{\beta}=\frac{k_{1}^{2} \beta}{g}
\end{aligned}
$$

Vol. $14, n^{\circ} 5-1997$. 
Equation (3.24) is the Davey-Stewartson system for the modulation of a solution to the water wave problem, with underlying spatial wavenumber $k$. Finally, the system (3.12) at order 3 can be solved in the form

$$
\begin{aligned}
\eta^{(3)}= & \delta_{1} e^{3} e^{3 i \varphi}+\delta_{2} c c_{z_{1}} e^{2 i \varphi} \\
& +\left(\delta_{3} c_{z_{1} z_{1}}+\delta_{4} c_{z_{2} z_{2}}+\delta_{5}|c|^{2} c+\delta_{6} c d_{z_{1}}\right) e^{i \varphi}+c . c+\delta_{7} \\
\xi^{(3)}= & \delta_{1}^{\prime} c^{3} e^{3 i \varphi}+\delta_{2}^{\prime} c c_{z_{1}} e^{2 i \varphi}+c . c
\end{aligned}
$$

with coefficients $\delta_{j}, \delta_{j}^{\prime}, j=1 \ldots 7$ depending on $\omega, k, h$ and $g$.

This establishes on a formal level that at leading order the interface deformation and the potential velocity on the surface have the form

$$
\begin{aligned}
& \eta=\epsilon \frac{i \omega}{g+\beta k_{1}^{2}} c\left(z_{1}, z_{2}, \tau\right) e^{i \varphi}+c . c \\
& \xi=\epsilon c\left(z_{1}, z_{2}, \tau\right) e^{i \varphi}+c . c+\epsilon d\left(z_{1}, z_{2}, \tau\right) .
\end{aligned}
$$

with $\varphi=k_{1} x_{1}-\omega t$ and $\omega^{2}=\left(g+\beta k_{1}^{2}\right) k_{1} \tanh \left(h k_{1}\right)$. The amplitude $c$ of the wave packet and the potential $d$ satisfy the Davey-Stewartson equations (3.24) in the slow variables $\tau=\epsilon^{2} t, z_{1}=\epsilon\left(x_{1}-\omega^{\prime} t\right)$ and $z_{2}=c x_{2}$. The coefficients $\lambda, \mu, \chi_{1}, \chi, \alpha$ and $\gamma$ depend on the gravity $g$, the depth $h$, the surface tension $\beta$ and the wave number $k_{1}$, as described in the above list.

\section{ANALYSIS OF THE DIRICHLET-NEUMANN OPERATOR IN THREE DIMENSIONS}

The purpose of this paper is to supply a rigorous basis for understanding the above formal procedure, which consists of several modulational scalings and the formal derivation of the Davey-Stewartson system (3.24). As posed in equations $(2.7)(2.8)$, the water wave equations form a nonlinear system of integro-differential equations in the free-surface variables $\left(x^{\prime}, t\right)$. In contrast, the Davey-Stewartson system is a nonlinear system of partial differential equations, and it is evident that the modulational approximations consist in part in approximating integral operators by partial differential operators. The central integral operator for the water wave problem is the DirichletNeumann operator $G(\eta)$. The analysis of this section is focused on the description of $G(\eta)$ and its dependence upon the fluid domain, through the function $\eta\left(x^{\prime}\right)$. The three basic facets of the analysis are (i) the analyticity of $G(\eta)$ in $\eta\left(x^{\prime}\right)$, and approximation of it by its Taylor series, (ii) the 
description of the Taylor remainder terms and their estimates, and (iii) the description of the individual terms in the Taylor series for $G(\eta)$ as pseudodifferential expressions, and estimates of their behavior under multiple scale expansions. The latter topic was addressed in a general setting in a previous paper [8]. We set about now to describe the former two.

\subsection{An exact implicit formula for $G$}

The fundamental solution of the Laplace equation in the domain $\left\{\mathbf{x} \in \mathbf{R}^{3}: x_{3}>-h\right\}$ which satisfies Neumann boundary condition at $x_{3}=-h$ is given by the method of images;

$$
\Gamma(\mathbf{x}, \mathbf{y})=-\frac{1}{4 \pi}\left(\frac{1}{|\mathbf{x}-\mathbf{y}|}+\frac{1}{\left|\mathbf{x}-\mathbf{y}^{*}\right|}\right), \quad \mathbf{x}, \mathbf{y} \in \mathbf{R}^{3}
$$

where $\mathbf{y}^{*}=\left(y^{\prime},-\left(2 h+y_{3}\right)\right)$ is the reflection of $y$ with respect to the bottom plane $x_{3}=-h$. For $\mathbf{x}=\left(x^{\prime}, \eta\left(x^{\prime}\right)\right)$ at the surface (with $x^{\prime} \in \mathbf{R}^{2}$ ) we denote the unit normal by

$$
N\left(x^{\prime}\right)=\left(1+\left(\partial_{x^{\prime}} \eta\right)^{2}\right)^{-1 / 2}\left(\begin{array}{c}
-\partial_{x^{\prime}} \eta \\
1
\end{array}\right) \text {. }
$$

Write the boundary values of a harmonic function $\varphi(\mathbf{x})$ as $\xi\left(x^{\prime}\right)=$ $\varphi\left(x^{\prime}, \eta\left(x^{\prime}\right)\right)$, and use Green's identity for a point $\left(x^{\prime}, \eta\left(x^{\prime}\right)\right)$ at the surface, to find

$$
\begin{aligned}
\frac{1}{2} \xi\left(x^{\prime}\right)=\frac{1}{2} \varphi\left(x^{\prime}, \eta\left(x^{\prime}\right)\right)= & \int_{\mathbf{R}^{2}} \xi\left(y^{\prime}\right) \partial_{y} \Gamma(\mathbf{x}, \mathbf{y}) \cdot N\left(y^{\prime}\right) \sqrt{1+\left(\partial_{y^{\prime}} \eta\right)^{2}} d y^{\prime} \\
& \int_{\mathbf{R}^{2}}(G(\eta) \xi)\left(y^{\prime}\right) \Gamma(\mathbf{x}, \mathbf{y}) d y^{\prime}
\end{aligned}
$$

where we are using the notation that $\mathbf{x}=\left(x^{\prime}, \eta\left(x^{\prime}\right)\right), \mathbf{y}=\left(y^{\prime}, \eta\left(y^{\prime}\right)\right)$. At the surface, we rewrite the Green's functions

$$
\begin{aligned}
\Gamma(\mathbf{x}, \mathbf{y})= & -\frac{1}{4 \pi}\left(\frac{1}{\left(\left|x^{\prime}-y^{\prime}\right|^{2}+\left(\eta\left(x^{\prime}\right)-\eta\left(y^{\prime}\right)\right)^{2}\right)^{1 / 2}}\right. \\
& \left.+\frac{1}{\left(\left|x^{\prime}-y^{\prime}\right|^{2}+\left(2 h+\eta\left(x^{\prime}\right)+\eta\left(y^{\prime}\right)\right)^{2}\right)^{1 / 2}}\right)
\end{aligned}
$$


and the double layer potential

$$
\begin{aligned}
& \sqrt{1+\left(\partial_{y^{\prime}} \eta\right)^{2}} \partial_{y} \Gamma(\mathbf{x}, \mathbf{y}) \cdot N\left(y^{\prime}\right) \\
& =\frac{1}{4 \pi}\left(\frac{\left(x^{\prime}-y^{\prime}\right) \cdot \partial_{y^{\prime}} \eta\left(y^{\prime}\right)-\left(\eta\left(x^{\prime}\right)-\eta\left(y^{\prime}\right)\right)}{\left(\left|x^{\prime}-y^{\prime}\right|^{2}+\left(\eta\left(x^{\prime}\right)-\eta\left(y^{\prime}\right)\right)^{2}\right)^{3 / 2}}\right. \\
& \left.+\frac{\left(x^{\prime}-y^{\prime}\right) \cdot \partial_{y^{\prime}} \eta\left(y^{\prime}\right)+\left(\eta\left(x^{\prime}\right)+\eta\left(y^{\prime}\right)+2 h\right)}{\left(\left|x^{\prime}-y^{\prime}\right|^{2}+\left(\eta\left(x^{\prime}\right)+\eta\left(y^{\prime}\right)+2 h\right)^{2}\right)^{3 / 2}}\right),
\end{aligned}
$$

which can be written as

$$
-\Gamma(x, y)=\frac{1}{4 \pi}\left(\frac{1}{\left|x^{\prime}-y^{\prime}\right|}+\frac{1}{\left(\left|x^{\prime}-y^{\prime}\right|^{2}+4 h^{2}\right)^{1 / 2}}\right)+\frac{1}{2} \ell\left(x^{\prime}, y^{\prime}\right)
$$

and

$$
\begin{aligned}
& \sqrt{1+\left(\partial_{y^{\prime}} \eta\right)^{2}} \partial_{y^{\prime}} \Gamma(x, y) \cdot N\left(y^{\prime}\right) \\
& =\frac{1}{4 \pi} \frac{2 h}{\left(\left|x^{\prime}-y^{\prime}\right|^{2}+4 h^{2}\right)^{3 / 2}}+\frac{1}{2} m\left(x^{\prime}, y^{\prime}\right)
\end{aligned}
$$

We denote the quotients in the above two expressions by

$$
\begin{aligned}
Q(\eta) & =\frac{\eta\left(x^{\prime}\right)-\eta\left(y^{\prime}\right)}{\left|x^{\prime}-y^{\prime}\right|} \\
Q_{1}(\eta) & =\frac{\eta\left(x^{\prime}\right)+\eta\left(y^{\prime}\right)}{\left(\left|x^{\prime}-y^{\prime}\right|^{2}+4 h^{2}\right)^{1 / 2}},
\end{aligned}
$$

obtaining the following expressions:

$$
\begin{aligned}
& \ell\left(x^{\prime}, y^{\prime}\right)=\frac{1}{2 \pi} \frac{1}{\left|x^{\prime}-y^{\prime}\right|}\left(\frac{1}{\left(1+Q^{2}\right)^{1 / 2}}-1\right) \\
& +\frac{1}{2 \pi} \frac{1}{\left(\left|x^{\prime}-y^{\prime}\right|^{2}+4 h^{2}\right)^{1 / 2}} \\
& \times\left(\frac{1}{\left(1+Q_{1} \frac{4 h}{\left(\left|x^{\prime} \cdots y^{\prime}\right|^{2}+4 h^{2}\right)^{1 / 2}}+Q_{1}^{2}\right)^{1 / 2}}-1\right) .
\end{aligned}
$$

Using the fact that

$$
\left(x^{\prime}-y^{\prime}\right) \cdot \partial_{y^{\prime}} Q=\frac{\eta\left(x^{\prime}\right)-\eta\left(y^{\prime}\right)-\left(x^{\prime}-y^{\prime}\right) \cdot \partial_{y^{\prime}} \eta\left(y^{\prime}\right)}{\left|x^{\prime}-y^{\prime}\right|}
$$


and

$$
\begin{array}{r}
\left(x^{\prime}-y^{\prime}\right) \cdot \partial_{y^{\prime}} Q_{1}+\frac{4 h^{2} Q_{1}}{\left|x^{\prime}-y^{\prime}\right|^{2}+4 h^{2}} \\
=\frac{\eta\left(x^{\prime}\right)+\eta\left(y^{\prime}\right)+\left(x^{\prime}-y^{\prime}\right) \cdot \partial_{y^{\prime}} \eta\left(y^{\prime}\right)}{\left(\left|x^{\prime}-y^{\prime}\right|^{2}+4 h^{2}\right)^{1 / 2}}
\end{array}
$$

the term $m\left(x^{\prime}, y^{\prime}\right)$ can be written in the following form.

$$
\begin{aligned}
m\left(x^{\prime}, y^{\prime}\right)= & -\frac{1}{2 \pi} \frac{\left(x^{\prime}-y^{\prime}\right) \cdot \partial_{y^{\prime}} Q}{\left|x^{\prime}-y^{\prime}\right|^{2}} \frac{1}{\left(1+Q^{2}\right)^{3 / 2}} \\
+ & \frac{1}{2 \pi}\left(\frac{\left(x^{\prime}-y^{\prime}\right) \cdot \partial_{y^{\prime}} Q_{1}}{\left(\left|x^{\prime}-y^{\prime}\right|^{2}+4 h^{2}\right)}+\frac{4 h^{2} Q_{1}}{\left(\left|x^{\prime}-y^{\prime}\right|^{2}+4 h^{2}\right)^{2}}\right) \\
& \times \frac{1}{\left.\left.\left(1+Q_{1} \frac{4 h}{\left(\left|x^{\prime}-y^{\prime}\right|^{2}+4 h^{2}\right)^{1 / 2}}+Q_{1}^{2}\right)\right)^{3 / 2}\right)} \\
+ & \frac{1}{2 \pi} \frac{2 h}{\left(\left|x^{\prime}-y^{\prime}\right|^{2}+4 h^{2}\right)^{3 / 2}} \\
& \times\left(\frac{1}{\left.\left(1+Q_{1} \frac{4 h}{\left(\left|x^{\prime}-y^{\prime}\right|^{2}+4 h^{2}\right)^{1 / 2}}+Q_{1}^{2}\right)\right)^{3 / 2}}-1\right)
\end{aligned}
$$

This will be used in the analysis of Section 4.2. We substitute these expressions in (4.2), obtaining

$$
\begin{aligned}
\xi\left(x^{\prime}\right)= & \frac{1}{2 \pi} \frac{2 h}{\left(\left|x^{\prime}\right|^{2}+4 h^{2}\right)^{3 / 2}} * \xi \\
& +\frac{1}{2 \pi}\left(\frac{1}{\left|x^{\prime}\right|}+\frac{1}{\left(\left|x^{\prime}\right|^{2}+4 h^{2}\right)^{1 / 2}}\right) * G(\eta) \xi \\
& +M(\eta) \xi+L(\eta) G(\eta) \xi
\end{aligned}
$$

with the two operators given by the above kernels,

$$
\begin{aligned}
M(\eta) \mu\left(x^{\prime}\right) & =\int m\left(x^{\prime}, y^{\prime}\right) \mu\left(y^{\prime}\right) d y^{\prime}, \\
L(\eta) \mu\left(x^{\prime}\right) & =\int \ell\left(x^{\prime}, y^{\prime}\right) \mu\left(y^{\prime}\right) d y^{\prime} .
\end{aligned}
$$

Denoting by $\mathcal{F}$ the Fourier transform operator, the convolution operators in the above expression are given explicitly by

$$
\mathcal{F}\left(\frac{1}{\left|x^{\prime}\right|}\right)=2 \pi|p|^{-1}
$$

Vol. 14, no 5-1997. 


$$
\mathcal{F}\left(\frac{1}{\left(\left|x^{\prime}\right|^{2}+4 h^{2}\right)^{1 / 2}}\right)=2 \pi|p|^{-1} e^{-2 h|p|}
$$

and

$$
\mathcal{F}\left(\frac{2 h}{\left(\left|x^{\prime}\right|^{2}+4 h^{2}\right)^{3 / 2}}\right)=2 \pi e^{-2 h|p|}
$$

(For completeness, we give a thesaurus in Appendix $\Lambda$.)

Equation (4.12) takes the form.

$$
\left(1-e^{-2 h|D|}\right) \xi-M(\eta) \xi=|D|^{-1}\left(1+e^{-2 h|D|}\right) G(\eta) \xi+L(\eta) G(\eta) \xi
$$

or equivalently:

$$
(1-B(\eta)) G(\eta) \xi=|D| \tanh (h|D|) \xi+A(\eta) \xi
$$

with

$$
B(\eta) \zeta=-\left(1+e^{-2 h|D|}\right)^{-1}|D| L(\eta) \zeta
$$

and

$$
A(\eta) \zeta=-\left(1+e^{-2 h|D|}\right)^{-1}|D| M(\eta) \zeta
$$

Identity (4.18) is the implicit form of the operator $G(\eta)$ that we will use. Since both $M(\eta)$ and $L(\eta)$ start at least linearly in $\eta,(4.18)$ gives directly that $G_{0}=|D| \tanh (h|D|)$.

\subsection{Frror terms in the Taylor expansion of $G$}

Starting from the implicit formulation of $G$ obtained in the previous section, we rederive the first three terms in the expansion of $G$ together with the explicit form of the error. Although this derivation is more complicated that the one presented in Section 2.2, it allows us to write precise estimates on the error terms.

The Taylor expansion of the kernel $\ell\left(x^{\prime}, y^{\prime}\right)$ defined in (4.8) is given by:

$$
\begin{aligned}
\ell\left(x^{\prime}, y^{\prime}\right)= & \frac{1}{2 \pi} \sum_{1 \leq j \leq J}\left(\frac{p_{j}(Q)}{|x-y|}+\frac{q_{j}\left(Q_{1}, \frac{4 h}{\left(\left|x^{\prime}-y^{\prime}\right|^{2}+4 h^{2}\right)^{1 / 2}}\right)}{\left(\left|x^{\prime}-y^{\prime}\right|^{2}+4 h^{2}\right)^{1 / 2}}\right) \\
& +\frac{1}{2 \pi} \frac{p_{J+1}^{R}(Q)}{\left|x^{\prime}-y^{\prime}\right|}+\frac{1}{2 \pi} \frac{q_{J+1}^{R}\left(Q_{1}, \frac{4 h}{\left(\left|x^{\prime}-y^{\prime}\right|^{2}+1 h^{2}\right)^{1 / 2}}\right)}{\left(\left|x^{\prime}-y^{\prime}\right|^{2}+4 h^{2}\right)^{1 / 2}}
\end{aligned}
$$


Using the relations (4.9)-(4.10), the Taylor expansion of the kernel $m$ takes the form

$$
\begin{aligned}
m\left(x^{\prime}, y^{\prime}\right)= & \frac{1}{2 \pi} \sum_{1 \leq j \leq J}\left(\frac{\left(x^{\prime}-y^{\prime}\right) \cdot \partial_{y^{\prime}} m_{j}(Q)}{\left|x^{\prime}-y^{\prime}\right|^{2}}\right. \\
& +\left(\frac{\left(x^{\prime}-y^{\prime}\right) \cdot \partial_{y^{\prime}} Q_{1}}{\left|x^{\prime}-y^{\prime}\right|^{2}+4 h^{2}}+\frac{4 h^{2} Q_{1}}{\left(\left|x^{\prime}-y^{\prime}\right|^{2}+4 h^{2}\right)^{2}}\right) \\
& \times n_{j-1}\left(Q_{1}, \frac{4 h}{\left(\left|x^{\prime}-y^{\prime}\right|^{2}+4 h^{2}\right)^{1 / 2}}\right) \\
+ & \left.\frac{1}{2 \pi} \frac{2 h}{\left(\left|x^{\prime}-y^{\prime}\right|^{2}+4 h^{2}\right)^{3 / 2}} n_{j}\left(Q_{1}, \frac{4 h}{\left(\left|x^{\prime}-y^{\prime}\right|^{2}+4 h^{2}\right)^{1 / 2}}\right)\right) \\
& +\frac{1}{2 \pi} \frac{\left(x^{\prime}-y^{\prime}\right) \cdot \partial_{y^{\prime}} m_{J+1}^{R}(Q)}{\left|x^{\prime}-y^{\prime}\right|^{2}} \\
+ & \frac{1}{2 \pi}\left(\frac{\left(x^{\prime}-y^{\prime}\right) \cdot \partial_{y^{\prime}} Q_{1}}{\left|x^{\prime}-y^{\prime}\right|^{2}+4 h^{2}}+\frac{4 h^{2} Q_{1}}{\left(\left|x^{\prime}-y^{\prime}\right|^{2}+4 h^{2}\right)^{2}}\right) \\
& \times n_{J-1}^{R}\left(Q_{1}, \frac{4 h}{\left(\left|x^{\prime}-y^{\prime}\right|^{2}+4 h^{2}\right)^{1 / 2}}\right) \\
& +\frac{1}{2 \pi} \frac{2 h}{\left(\left|x^{\prime}-y^{\prime}\right|^{2}+4 h^{2}\right)^{3 / 2}} n_{J}^{R}\left(Q_{1}, \frac{4 h}{\left(\left|x^{\prime}-y^{\prime}\right|^{2}+4 h^{2}\right)^{1 / 2}}\right) .
\end{aligned}
$$

The functions $p_{j}, q_{j}, m_{j}$ and $n_{j}$ are homogeneous polynomials of degree $j$ in $\eta\left(x^{\prime}\right)$ and $\eta\left(y^{\prime}\right)$. The expressions $p_{J}^{R}, q_{J}^{R}, m_{J}^{R}, n_{J}^{R}$ are Taylor remainders that come from Taylor expansions of $(1+\sigma)^{-1 / 2}$ and similar expressions, so that $p_{L}^{R}(\sigma) \sim \mathcal{O}\left(\sigma^{L}\right)$ for small $\sigma$, and analogous estimates hold for the other quantities in their arguments.

Using (4.20) and (4.21), we write

with

$$
\begin{aligned}
& A(\eta)=A_{1}(\eta)+\cdots+A_{J}(\eta)+A_{J}^{R} \\
& B(\eta)=B_{1}(\eta)+\cdots+B_{J}(\eta)+B_{J}^{R},
\end{aligned}
$$

$$
\begin{aligned}
A_{j}(\eta) \xi=- & |D|\left(1+e^{-2 h|D|}\right)^{-1} \frac{1}{2 \pi}\left(\int\left(\frac{\left(x^{\prime}-y^{\prime}\right) \cdot \partial_{y^{\prime}} m_{j}(Q)}{\left|x^{\prime}-y^{\prime}\right|^{2}}\right) \xi\left(y^{\prime}\right) d y^{\prime}\right. \\
+ & \frac{1}{2 \pi} \int\left(\frac{\left(x^{\prime}-y^{\prime}\right) \cdot \partial_{y^{\prime}} Q_{1}}{\left|x^{\prime}-y^{\prime}\right|^{2}+4 h^{2}}+\frac{4 h^{2} Q_{1}}{\left(\left|x^{\prime}-y^{\prime}\right|^{2}+4 h^{2}\right)^{2}}\right) \\
& \times n_{j-1}\left(Q_{1}, \frac{4 h}{\left(\left|x^{\prime}-y^{\prime}\right|^{2}+4 h^{2}\right)^{1 / 2}}\right) \xi\left(y^{\prime}\right) d y^{\prime} \\
+ & \frac{1}{2 \pi} \int \frac{2 h}{\left(\left|x^{\prime}-y^{\prime}\right|^{2}+4 h^{2}\right)^{3 / 2}} \\
& \left.\times n_{j}\left(Q_{1}, \frac{4 h}{\left(\left|x^{\prime}-y^{\prime}\right|^{2}+4 h^{2}\right)^{1 / 2}}\right) \xi\left(y^{\prime}\right) d y^{\prime}\right)
\end{aligned}
$$


and

$$
\begin{aligned}
B_{j}(\eta) \xi= & -|D|\left(1+e^{-2 h|D|}\right)^{-1} \\
& \times \frac{1}{2 \pi} \int\left(\frac{p_{j}(Q)}{\left|x^{\prime}-y^{\prime}\right|}+\frac{q_{j}\left(Q_{1}, \frac{4 h}{\left(\left|x^{\prime}-y^{\prime}\right|^{2}+4 h^{2}\right)^{1 / 2}}\right)}{\left(\left|x^{\prime}-y^{\prime}\right|^{2}+4 h^{2}\right)^{1 / 2}}\right) \xi\left(y^{\prime}\right) d y^{\prime} .
\end{aligned}
$$

We denote by $A_{J}^{R}$ and $B_{J}^{R}$ the terms which describe the Taylor remainder. For our purposes, we need to study the third order expansion. From (4.18), we gel

$$
\begin{aligned}
G(\eta) \xi= & G_{0} \xi+\left(B_{1} G_{0}+A_{1}\right) \xi \\
& +\left(B_{2} G_{0}+B_{1} A_{1}+A_{2}+B_{1}^{2} G_{0}\right) \xi+R_{3}(\eta) \xi
\end{aligned}
$$

where the remainder $R_{3}(\eta)$ has the form

$$
\begin{aligned}
R_{3}(\eta)= & \left(B_{3}^{R}+B_{1} B_{2}+B_{2} B_{1}+B_{1} B_{3}^{R}+B_{3}^{R} B_{1}+B_{2} B_{3}^{R}\right. \\
& \left.+B_{3}^{R} B_{2}+\left(B_{3}^{R}\right)^{2}+B_{2}^{2}\right) G_{0}+\left(B_{2}+B_{3}^{R}\right) A_{1} \\
& +(1-B)^{-1}\left(A_{3}^{R}+B A_{2}+B^{2} A_{1}+B^{3} G_{0}\right) .
\end{aligned}
$$

THEOREM 4.1. - The Dirichlet-Neumann operator can be written in the form:

$$
G(\eta)=G_{0}+G_{1}(\eta)+G_{2}(\eta)+R_{3}(\eta)
$$

with

$$
\begin{aligned}
G_{0} & =|D| \tanh (h|D|) \\
G_{1} & =D \cdot \eta D-G_{0} \eta G_{0} \\
G_{2} & =-\frac{1}{2}|D|^{2} \eta^{2} G_{0}+G_{0} \eta G_{0} \eta G_{0}-\frac{1}{2} G_{0} \eta^{2}|D|^{2}
\end{aligned}
$$

with the remainder $R_{3}(\eta)$ defined in (4.26).

Proof of Theorem 4.1. - We will derive the explicit form of the operators $A_{1}, A_{2}, B_{1}, B_{2}$, in order to express $G_{1}, G_{2}$

$$
\begin{aligned}
A_{1}(\eta) \xi= & -|D|\left(1+e^{-2 h|D|}\right)^{-1} \\
& \times \frac{1}{2 \pi} \int\left(\frac{\left(x^{\prime}-y^{\prime}\right) \cdot \partial_{y^{\prime}} \eta\left(y^{\prime}\right)-\left(\eta\left(x^{\prime}\right)-\eta\left(y^{\prime}\right)\right)}{\left|x^{\prime}-y^{\prime}\right|^{3}}\right. \\
& +\frac{\left(x^{\prime}-y^{\prime}\right) \cdot \partial_{y^{\prime}} \eta\left(y^{\prime}\right)+\eta\left(x^{\prime}\right)+\eta\left(y^{\prime}\right)}{\left(\left|x^{\prime}-y^{\prime}\right|^{2}+4 h^{2}\right)^{3 / 2}} \\
& \left.-\frac{12 h^{2} Q_{1}}{\left(\left|x^{\prime}-y^{\prime}\right|^{2}+4 h^{2}\right)^{2}}\right) d y^{\prime}
\end{aligned}
$$


We can write these expressions in terms of Fourier multipliers (see Appendix A)

$$
\begin{aligned}
A_{1}(\eta) \xi & =D \cdot \eta D \xi-|D|\left(1+e^{-2 h|D|}\right)^{-1} \eta|D|\left(1-e^{-2 h|D|}\right) \xi \\
& =D \cdot \eta D \xi-|D|\left(1+e^{2 h|D|}\right)^{-1} \eta\left(1+e^{-2 h|D|}\right) G_{0} \xi .
\end{aligned}
$$

Similarly,

$$
\begin{aligned}
B_{1}(\eta) \xi= & -|D|\left(1+e^{-2 h|D|}\right)^{-1} \\
& \times\left(-\frac{1}{2 \pi}\right) \int \frac{2 h}{\left(\left|x^{\prime}-y^{\prime}\right|^{2}+4 h^{2}\right)^{3 / 2}}\left(\eta\left(x^{\prime}\right)+\eta\left(y^{\prime}\right)\right) \xi\left(y^{\prime}\right) d y^{\prime} \\
= & |D|\left(1+e^{-2 h|D|}\right)^{-1}\left(\eta e^{-2 h|D|} \xi+e^{-2 h|D|} \eta \xi\right) .
\end{aligned}
$$

Thus, combining (4.30) and (4.31), we get

$$
\begin{aligned}
G_{1}(\eta) \xi & =\left(B_{1} G_{0}+A_{1}\right) \xi \\
& =\left(D \cdot \eta D-G_{0} \eta G_{0}\right) \xi
\end{aligned}
$$

For $G_{2}$, we have

$$
G_{2}(\eta) \xi=\left(B_{2} G_{0}+B_{1} A_{1}+A_{2}+B_{1}^{2} G_{0}\right) \xi
$$

with

$$
\begin{aligned}
A_{2}(\eta) \xi= & -|D|\left(1+e^{-2 h|D|}\right)^{-1} \\
& \times \frac{1}{2 \pi} \int\left((-6 h) \frac{\left(x^{\prime}-y^{\prime}\right) \cdot \partial_{y^{\prime}} \eta\left(y^{\prime}\right)+\eta\left(x^{\prime}\right)+\eta\left(y^{\prime}\right)}{\left(\left|x^{\prime}-y^{\prime}\right|^{2}+4 h^{2}\right)^{2}} Q_{1}\right. \\
& \left.-\frac{3 h Q_{1}^{2}}{\left(\left|x^{\prime}-y^{\prime}\right|^{2}+4 h^{2}\right)^{3 / 2}}\left(1-\frac{20 h^{2}}{\left|x^{\prime}-y^{\prime}\right|^{2}+4 h^{2}}\right)\right) \xi\left(y^{\prime}\right) d y^{\prime} \\
= & -|D|\left(1+e^{-2 h|D|}\right)^{-1}\left(\eta i D \cdot e^{-2 h|D|}\left(\left(\partial_{y^{\prime}} \eta\right) \xi\right)\right. \\
& +i D \cdot e^{-2 h|D|}\left(\left(\partial_{y^{\prime}} \eta\right) \eta \xi\right)-\frac{3}{8 h^{2}}\left(\eta^{2}(1+2 h|D|) e^{-2 h|D|}\right. \\
& \left.+2 \eta(1+2 h|D|) e^{-2 h|D|} \eta+(1+2 h|D|) e^{-2 h|D|} \eta^{2}\right) \xi \\
& +\frac{15}{4 h^{2}}\left(\eta^{2}\left(\frac{1}{10}(1+2 h|D|)+\frac{2}{15} h^{2}|D|^{2}\right) e^{-2 h|D|} \xi\right. \\
& +2 \eta\left(\frac{1}{10}(1+2 h|D|)+\frac{2}{15} h^{2}|D|^{2}\right) \eta e^{-2 h|D|} \xi \\
& \left.\left.+\left(\frac{1}{10}(1+2 h|D|)+\frac{2}{15} h^{2}|D|^{2}\right) e^{-2 h|D|} \eta^{2} \xi\right)\right) \\
= & -|D|\left(1+e^{-2 h|D|}\right)^{-1}\left(\eta D \cdot e^{-2 h|D|} \eta D\right. \\
& \left.+\frac{1}{2} D \cdot e^{-2 h|D|} \eta^{2} D+\frac{1}{2} \eta^{2}|D|^{2} e^{-2 h|D|}\right) \xi
\end{aligned}
$$




$$
\begin{aligned}
B_{2}(\eta) \xi= & |D|\left(1+e^{-2 h|D|}\right)^{-1} \frac{1}{2 \pi} \int\left(\frac{1}{2\left|x^{\prime}-y^{\prime}\right|^{3}}\left(\eta\left(x^{\prime}\right)-\eta\left(y^{\prime}\right)\right)^{2}\right. \\
& +\frac{1}{2\left(\left|x^{\prime}-y^{\prime}\right|^{2}+4 h^{2}\right)^{3 / 2}}\left(\eta\left(x^{\prime}\right)+\eta\left(y^{\prime}\right)\right)^{2} \\
& \left.-\frac{6 h^{2}}{\left(\left|x^{\prime}-y^{\prime}\right|^{2}+4 h^{2}\right)^{5 / 2}}\left(\eta\left(x^{\prime}\right)+\eta\left(y^{\prime}\right)\right)^{2}\right) \xi d y^{\prime} \\
= & -|D|\left(1+e^{-2 h|D|}\right)^{-1} \frac{1}{2}\left(\eta^{2}|D|\left(1+e^{-2 h|D|}\right)\right. \\
& \left.+2 \eta|D|\left(-1+e^{-2 h|D|}\right) \eta+|D|\left(1+e^{-2 h|D|}\right) \eta^{2}\right) \xi
\end{aligned}
$$

The expressions that we seek are given by

$$
\begin{aligned}
B_{2} G_{0} \xi= & -|D|\left(1+e^{-2 h|D|}\right)^{-1} \frac{1}{2} \eta^{2}|D|^{2}\left(1-e^{-2 h|D|}\right) \xi \\
- & |D|\left(1+e^{-2 h|D|}\right)^{-1} \eta|D|\left(-1+e^{-2 h|D|}\right) \eta G_{0} \xi \\
- & \frac{1}{2}|D|^{2} \eta^{2} G_{0} \xi \\
\left(B_{1} A_{1}+B_{1}^{2} G_{0}\right) \xi= & B_{1} G_{1} \xi \\
= & |D|\left(1+e^{-2 h|D|}\right)^{-1}\left(\eta e^{-2 h|D|}+e^{-2 h|D|} \eta\right) \\
& \times\left(D \cdot \eta D-G_{0} \eta G_{0}\right) \xi
\end{aligned}
$$

and $A_{2} \xi$ is given in (4.34).

Now we combine these 3 cxpressions and get

$$
\begin{aligned}
\left(B_{2} G_{0}\right. & \left.+A_{2}+B_{1} A_{1}+B_{1}^{2} G_{0}\right) \xi \\
= & -G_{0} \frac{1}{2} \eta^{2}|D|^{2} \xi-|D|\left(1+e^{-2 h|D|}\right)^{-1} e^{-2 h|D|} \frac{1}{2} \eta^{2}|D|^{2} \xi \\
& +|D|\left(1+e^{-2 h|D|}\right)^{-1} \eta G_{0}\left(1+e^{-2 h|D|}\right) \eta G_{0} \xi-|D|^{2} \frac{1}{2} \eta^{2} G_{0} \xi \\
& -|D|\left(1+e^{-2 h|D|}\right)^{-1}\left(\eta e^{-2 h|D|} D \cdot \eta D \xi+e^{-2 h|D|} D \cdot \frac{1}{2} \eta^{2} D \xi\right) \\
& +|D|\left(1+e^{-2 h|D|}\right)^{-1}\left(\eta e^{-2 h|D|}+e^{-2 h|D|} \eta\right) \\
& \times\left(D \cdot \eta D-G_{0} \eta G_{0}\right) \xi .
\end{aligned}
$$

A simple commutator identity gives that

$$
-\frac{1}{2} \eta^{2}|D|^{2} \xi+\eta D \cdot(\eta D \xi)-D \cdot \frac{1}{2} \eta^{2} D \xi=0
$$

so that we obtain the desired expression;

$$
G_{2} \xi=-G_{0} \frac{1}{2} \eta^{2}|D|^{2} \xi-|D|^{2} \frac{1}{2} \eta^{2} G_{0} \xi+G_{0} \eta G_{0} \eta G_{0}
$$

The next section gives precise estimates on the error term $R_{3}(\eta)$ defined in (4.27). 


\subsection{Analyticity of the Dirichlet-Neumann operator}

In this section, we prove the analyticity of $G$ as an operator between appropriate Sobolev spaces, for $\eta$ in a neighborhood $|\eta|_{C^{1}}<R_{0}$. A related result was proved by Coifman and Meyer [4] in one space dimension for $\eta$ with bounded Lipschitz norm $\left|\partial_{x^{\prime}} \eta\right|_{\infty}$ (that is, for $\left(x^{\prime}, \eta\left(x^{\prime}\right)\right)$ a Lipschitz graph in $\mathbf{R}^{2}$ ). Here $\left(x^{\prime}, \eta\left(x^{\prime}\right)\right)$ is a surface in $\mathbf{R}^{3}$, and the absence of the use of complex variables makes the theorem more complicated. The main consequence of the present result is an estimate for the Taylor remainder term $R_{3}(\eta)$ resulting from the expansion of $G(\eta)$ to second order (see (eq. (4.28)).

We use the notation $W^{s, q}\left(\mathbf{R}^{n}\right)$ for the Sobolev space with the norm $\|u\|_{s, q}^{q}=\sum_{|m| \leq s}\left\|\partial_{x^{\prime}}^{m} u\right\|_{q}^{q}$, where $\|\cdot\|_{q}$ denotes the usual norm on $L^{q}\left(\mathbf{R}^{n}\right)$. We also use the space $C^{s}\left(\mathbf{R}^{n}\right)$ for the continuous functions whose derivative functions up to order $s$ are also continuous, with the usual norm $|\eta|_{C^{s}}=\sum_{|\ell|<s}\left|\partial_{x^{\prime}}^{\ell} \eta\right|_{L^{\infty}}$.

TheOrem 4.2. - Let $1<q<+\infty$. There is a constant $R_{0}$ such that the operator $G(\eta)$ is analytic in $\eta$ in the neighborhood $\left\{\eta:|\eta|_{C^{1}}<R_{0}\right.$, $\left.|\eta|_{C^{s+1}}<\infty\right\}$, as a mapping $G(\eta): W^{s+1, q} \rightarrow W^{s, q}$.

We first focus on the operators $A$ and $B$ expressed in (4.22) and (4.23)(4.24), as the theorem will follow from a series of results for them. The effort in this section is to reduce these operators to a standard form as singular integral operators. The conclusions of this section will then follow from the $L^{q}$ and $W^{s, q}$ mapping properties of these operators, which is the focus of the analysis of Section 5 of this paper. The general form of these singular integral operators is

$$
C_{p}(\eta) \xi\left(x^{\prime}\right)=\int k\left(x^{\prime}-y^{\prime}\right) c_{p}(Q) \xi\left(y^{\prime}\right) d y^{\prime},
$$

where $k$ is a convolution operator of Calderón-Zygmund type and $c_{p}(\sigma)$ is an analytic function in a neighborhood of the origin which satisfies $c_{p}(\sigma) \sim O\left(\sigma^{p}\right)$. It is also necessary to study related smoothing operators of the form

$$
\begin{aligned}
& C_{p, h}(\eta) \xi\left(x^{\prime}\right) \\
& \quad=\int k_{h}\left(x^{\prime}-y^{\prime}\right) c_{p, h}\left(Q_{1}, \frac{4 h}{\left(\left|x^{\prime}-y^{\prime}\right|^{2}+4 h^{2}\right)^{1 / 2}}\right) \xi\left(y^{\prime}\right) d y^{\prime},
\end{aligned}
$$

where $k_{h}$ is in a particular class of smoothing kernels and $c_{p, h}(\sigma, \tau)$ is an analytic function in a neighborhood of the origin that satisfies $c_{p, h} \sim O\left(\sigma^{p} \tau^{r}\right)$, with $r \geq 1$.

Vol. $14, \mathbf{n}^{\circ}$ 5-1997. 
THEOREM 4.3. - Let $1<q<+\infty$, and $0 \leq s \in \mathbf{Z}$. The operators $B_{J}$ and $B_{J}^{R}$ defined in (4.22)-(4.24) satisfy the estimate:

(i) $\left\|B_{J}(\eta) \xi\right\|_{s, q} \leq|\eta|_{C^{1}}^{J-1}\left(C(s)|\eta|_{C^{s+1}}\|\xi\|_{q}+|\eta|_{C^{1}}\|\xi\|_{s, q}\right)$

(ii) $\left\|B_{J}^{R}(\eta) \xi\right\|_{s, q} \leq|\eta|_{C^{1}}^{J-1}\left(C(s)|\eta|_{C^{s+1}}\|\xi\|_{q}+|\eta|_{C^{1}}\|\xi\|_{s, q}\right)$

COROllary 4.4

(i) The powers of $B$ satisfy

$\left\|B^{j}(\eta) \xi\right\|_{s, q} \leq|\eta|_{C^{1}}^{j-1}\left(|\eta|_{C^{1}}\|\xi\|_{s, q}+C(s)\left(1+C_{0}\right)^{j-1}|\eta|_{C^{s+1}}\|\xi\|_{q}\right)$

(ii) For $|\eta|_{C^{1}}$ small enough, the operator $(1-B)$ is invertible and satisfies

$$
\left\|(1-B)^{-1} \xi\right\|_{s, q} \leq 1+\left(C(s)|\eta|_{C^{s+1}}\|\xi\|_{q}+|\eta|_{C^{1}}\|\xi\|_{s, q}\right)
$$

Proof of Theorem 4.3. - First of all, we notice that the operator $\left(1+e^{-2 h|D|}\right)^{-1}$ is bounded in $W^{s, q}$ for all $1<q<+\infty$ and all $s \geq 0$, and furthermore

$$
|D|=-\sum_{j=1}^{n} i D_{j}|D|^{-1} \cdot i D_{j}=-\sum_{j=1}^{n} R_{j}(D) \partial_{x^{\prime} j}
$$

where $R_{j}(D)$ are the standard Riesz potentials which are also $L^{q}$-bounded. Thus, to find a bound for $B_{j}$ defined in (4.24), we are led to consider two types of integral operators $P_{j}$ and $Q_{j, h}$ defined by

$$
P_{j}(\eta) \xi=\partial_{x_{i}^{\prime}} \int \frac{1}{\left|x^{\prime}-y^{\prime}\right|} p_{j}(Q(\eta)) \xi\left(y^{\prime}\right) d y^{\prime}
$$

where $p_{j}(Q)=a_{j} Q^{j}$

$$
\begin{aligned}
\left.Q_{j, h}(\eta) \xi=\partial_{x_{i}^{\prime}} \int \frac{1}{\left(\left|x^{\prime}-y^{\prime}\right|^{2}\right.}+4 h^{2}\right)^{1 / 2} & \\
& \times q_{j}\left(Q_{1}(\eta), \frac{4 h}{\left(\left|x^{\prime}-y^{\prime}\right|^{2}+4 h^{2}\right)^{1 / 2}}\right) \xi\left(y^{\prime}\right) d y^{\prime}
\end{aligned}
$$

We rewrite $P_{j}$ in the form:

$$
\begin{aligned}
P_{j}(\eta) \xi=a_{j} & \left(\int \frac{x_{i}^{\prime}-y_{i}^{\prime}}{\left|x^{\prime}-y^{\prime}\right|^{3}} Q^{j} \xi\left(y^{\prime}\right) d y^{\prime}\right. \\
& \left.+\partial_{x_{i}^{\prime}} \eta\left(x^{\prime}\right) \int \frac{1}{\left|x^{\prime}-y^{\prime}\right|^{2}} Q^{j-1} \xi\left(y^{\prime}\right) d y^{\prime}\right)
\end{aligned}
$$


which is a sum of operators $C_{j}(\eta)$ defined in (4.40) with kernel

$$
k(x-y)=\frac{x_{i}^{\prime}-y_{i}^{\prime}}{|x-y|^{3}} \quad \text { and } \quad \partial_{x_{i}^{\prime}} \eta\left(x^{\prime}\right) C_{j-1}(\eta)
$$

which involves the kernel $k\left(x^{\prime}-y^{\prime}\right)=\left(1 /\left|x^{\prime}-y^{\prime}\right|^{2}\right)$.

Similarly, and less delicately, we may write

$$
\begin{aligned}
Q_{j, h}(\eta) \xi= & \sum b_{\gamma}^{(1)} \int \frac{(4 h)^{\gamma_{2}}\left(x_{i}^{\prime}-y_{i}^{\prime}\right)}{\left(\left|x^{\prime}-y^{\prime}\right|^{2}+4 h^{2}\right)^{\gamma_{1}}} Q_{1}^{j}(\eta) \xi\left(y^{\prime}\right) d y^{\prime} \\
+ & \sum b_{\gamma}^{(2)} \partial_{x_{i}^{\prime}} \eta\left(x^{\prime}\right) \int \frac{(4 h)^{\gamma_{4}}}{\left(\left|x^{\prime}-y^{\prime}\right|^{2}+4 h^{2}\right)^{\gamma_{3}}} \\
& \times Q_{1}^{j-1}(\eta) \xi\left(y^{\prime}\right) d y^{\prime}
\end{aligned}
$$

with coefticients derived from the binomial expansion. These are clearly in the form of operators of type $C_{j, h}(\eta)$ with smoothing kernels $k_{h}\left(x^{\prime}-y^{\prime}\right)=$ $\left(1 /\left(\left|x^{\prime}-y^{\prime}\right|^{2}+4 h^{2}\right)^{\gamma_{1}}\right)$ or $k_{h}(x-y)=\left(\left(x_{i}^{\prime}-y_{i}^{\prime}\right) /\left(\left|x^{\prime}-y^{\prime}\right|^{2}+4 h^{2}\right)^{\gamma_{2}}\right)$.

We have achieved the form for which the $L^{q}$ and $W^{s, q}$ transformation properties of singular integral operators may be used. Applying Theorem 5.7 to the operators $P_{j}(\eta)$ and Theorem 5.8 to $Q_{j, h}(\eta)$ of $B_{j}(\eta)$, we see that the estimates (4.42) hold. The operators $B_{J}^{R}(\eta)$ do not have kernel which are homogeneous polynomials in $Q, Q_{1}$, but rather analytic functions of $Q$ and $Q_{1}$, and the corresponding result (4.43) follows from Theorems 5.1 and 5.2 respectively.

Proof of Corollary 4.4. - Noting that $B=B_{1}^{R}$, we have the estimate that

$$
\|B(\eta) \xi\|_{s, q} \leq C(s)|\eta|_{C^{s+1}}\|\xi\|_{q}+|\eta|_{C^{1}}\|\xi\|_{s, q}
$$

To prove estimate (4.44), we will proceed by induction. Suppose that

$$
\left\|B^{j}(\eta) \xi\right\|_{s, q} \leq|\eta|_{C^{1}}^{j}\|\xi\|_{s, q}+C(s)\left(1+C_{0}\right)^{j-1}|\eta|_{C^{1}}^{j-1}|\eta|_{C^{s+1}}\|\xi\|_{q}
$$

and let us prove that the same estimate holds for $B^{j+1}(\eta)$. Applying (4.51) to $B(\eta) \zeta$, we have:

$$
\begin{aligned}
\left\|B^{j+1}(\eta) \xi\right\|_{s, q} \leq & |\eta|_{C^{1}}^{j-1}\left(|\eta|_{C^{1}}\|B(\eta) \xi\|_{s, q}\right. \\
& \left.+\left(1+C_{0}\right)^{j-1} C(s)|\eta|_{C^{s+1}}\|B(\eta) \xi\|_{q}\right) \\
\leq & |\eta|_{C^{1}}^{j-1}\left(|\eta|_{C^{1}}\left(|\eta|_{C^{1}}\|\xi\|_{s, q}+C(s)|\eta|_{C^{s+1}}|\eta|_{C^{1}}\|\xi\|_{q}\right)\right. \\
& \left.+\left(1+C_{0}\right)^{j-1} C(s)|\eta|_{C^{s+1}} C_{0}|\eta|_{C^{1}}\|\xi\|_{q}\right) \\
\leq & |\eta|_{C^{1}}^{j}\left(|\eta|_{C^{1}}\|\xi\|_{s, q}+C(s)\left(1+C_{0}\right)^{j}|\eta|_{C^{s+1}}\|\xi\|_{q}\right)
\end{aligned}
$$

and estimate (4.44) on $B^{j}$ is complete.

Vol. $14, n^{\circ}$ 5-1997. 
Within the region of convergence of the geometric series $\sum_{j}|\eta|_{C^{1}}^{j}$ and $\sum_{j}\left(1+C_{0}\right)^{j-1}|\eta|_{C^{1}}^{j-1}$, that is for $|\eta|_{C^{1}}<1 /\left(1+C_{0}\right)$, we have convergence of the Neumann series for $(1-B)^{-1}$. This is our first condition on $|\eta|_{C^{1}}$, that $R_{0}<1 /\left(1+C_{0}\right)$. We now turn to the family of operators $A_{j}(\eta)$. To obtain the correct dependence on the smoothness of $\eta(x)$, we will use the following general lemma.

Lemma 4.5. - For $x \in \mathbf{R}^{n}, R(Q)$ an odd continuous function of $Q$ and $\eta \in C^{1}\left(\mathbf{R}^{n}\right)$, we have

$$
\int \frac{x-y}{|x-y|^{n}} \cdot \partial_{y}(R(Q)) \xi(y) d y=-\int \frac{x-y}{|x-y|^{n}} R(Q) \cdot \partial_{y} \xi(y) d y .
$$

Proof of Lemma 4.5. - Denote $B_{\epsilon}(x)$ a ball in $\mathbf{R}^{n}$ of radius $\epsilon$ and center $x$ and $S_{\epsilon}(x)$ its boundary. Then, for any $\eta, \xi \in C^{1}\left(\mathbf{R}^{n}\right)$,

$$
\begin{aligned}
\int_{\mathbf{R}^{n}-B_{c}} & \frac{x-y}{|x-y|^{n}} \cdot \partial_{y} R(Q) \xi(y) d y \\
= & -\int_{\mathbf{R}^{n}-B_{c}} \frac{x-y}{|x-y|^{n}} R(Q) \partial_{y} \xi(y) d y \\
& -\int_{\mathbf{R}^{n}-B_{c}} \partial_{y} \cdot\left(\frac{x-y}{|x-y|^{n}}\right) R(Q) \xi(y) d y \\
& +\int_{S_{,}(x)} \frac{x-y}{|x-y|^{n}} \cdot \frac{x-y}{|x-y|} R(Q) \xi(y) d S_{y}
\end{aligned}
$$

The second term of the r.h.s of (4.54) vanishes, as the vector field is divergence free in $\mathbf{R}^{n}-B_{\epsilon}$. Consider now the third term in (4.54). For $\xi$ continuous the difference between this term and

$$
\int_{S_{(}(x)} \frac{1}{|x-y|^{n-1}} R(Q) \xi(x) d S_{y}
$$

is of order $O(\epsilon)$, hence it suffices to consider the latter. By the mean value theorem,

$$
\begin{aligned}
& \int_{S_{\varepsilon}(x)} \frac{1}{|x-y|^{n-1}} R\left(\frac{\eta(x)-\eta(y)}{|x-y|}\right) d S_{y} \\
& \quad=\int_{S_{\mathrm{t}}(x)} \frac{1}{|x-y|^{n-1}} R\left(\partial_{x} \eta(z) \cdot \frac{x-y}{|x-y|}\right) d S_{y}
\end{aligned}
$$

for some $z=\tau x+(1-\tau) y, 0<\tau<1$. Assuming that $\partial_{x} \eta$ is continuous, it suffices to consider the following integral

$$
\int_{S_{\epsilon}(x)} \frac{1}{|x-y|^{n-1}} R\left(\partial_{x} \eta(x) \cdot \frac{x y}{|x-y|}\right) d S_{y}
$$


as the difference between (4.55) and (4.56) is again $O(\epsilon)$. However the integral in (4.56) vanishes, as $R(\cdot)$ is odd, as is its argument.

Remark. - This argument uses the fact that $\eta \in C^{1}\left(\mathbf{R}^{n}\right)$ is an essential way, and will not extend directly to $\eta \in \operatorname{Lip}\left(\mathbf{R}^{n}\right)$. However by an approximation argument it is easy to see that we may take $\xi \in W^{s, q}\left(\mathbf{R}^{n}\right)$.

Theorem 4.6. - The operators $A_{J}$ and $A_{J}^{R}$ defined in (4.23) and (4.25) satisfy the estimates:

(i) $\left\|A_{J}(\eta) \xi\right\|_{s, q} \leq C(s)|\eta|_{C^{1}}^{J-1}\left(|\eta|_{C^{s+1}}\left\|\partial_{x^{\prime}} \xi\right\|_{q}+|\eta|_{C^{1}}\left\|\partial_{x^{\prime}} \xi\right\|_{s, q}\right)$

(ii) $\left\|A_{J}^{R}(\eta) \xi\right\|_{s, q} \leq C(s)|\eta|_{C^{1}}^{J-1}\left(|\eta|_{C^{s+1}}\left\|\partial_{x^{\prime}} \xi\right\|_{q}+|\eta|_{C^{1}}\left\|\partial_{x^{\prime}} \xi\right\|_{s, q}\right)$

Proof of Theorem 4.6. - Using Lemma 4.5, the problem is transformed to a situation very similar to that of Theorem 4.3 . We have

$$
\begin{aligned}
A_{j}(\eta) \xi\left(x^{\prime}\right)= & -\frac{1}{2 \pi}\left(1+e^{-2 h|D|}\right)^{-1} \sum_{i} R_{i}(D) \\
& \times\left(\partial_{x^{\prime}{ }_{i}} \int \frac{x^{\prime}-y^{\prime}}{\left|x^{\prime}-y^{\prime}\right|^{2}} \cdot \partial_{y} m_{j}(Q) \xi\left(y^{\prime}\right) d y^{\prime}\right. \\
& +\partial_{x^{\prime}{ }_{i}} \int\left(\frac{\left(x^{\prime}-y^{\prime}\right) \cdot \partial_{y^{\prime}} Q_{1}}{\left|x^{\prime}-y^{\prime}\right|^{2}+4 h^{2}}+\frac{4 h^{2} Q_{1}}{\left(\left|x^{\prime}-y^{\prime}\right|^{2}+4 h^{2}\right)^{2}}\right) \\
& \times n_{j-1}\left(Q_{1}, \frac{4 h}{\left(\left|x^{\prime}-y^{\prime}\right|^{2}+4 h^{2}\right)^{1 / 2}}\right) \xi\left(y^{\prime}\right) d y^{\prime} \\
& +\partial_{x^{\prime}{ }_{i}} \int \frac{2 h}{\left(\left|x^{\prime}-y^{\prime}\right|^{2}+4 h^{2}\right)^{3 / 2}} \\
& \left.\times n_{j}\left(Q_{1}, \frac{4 h}{\left(\left|x^{\prime}-y^{\prime}\right|^{2}+4 h^{2}\right)^{1 / 2}}\right) \xi\left(y^{\prime}\right) d y^{\prime}\right)
\end{aligned}
$$

We use Lemma 4.5 to rewrite the relevant part as a sum of operators of the two forms. The most sensitive terms are the singular integrals

$$
\begin{aligned}
P_{j}(\eta) \xi\left(x^{\prime}\right)= & -\partial_{x^{\prime}{ }_{i}} \int \frac{x^{\prime}-y^{\prime}}{\left|x^{\prime}-y^{\prime}\right|^{2}} \cdot m_{j}(Q) \partial_{y^{\prime}} \xi\left(y^{\prime}\right) d y^{\prime} \\
= & -\int \frac{1}{\left|x^{\prime}-y^{\prime}\right|^{2}} m_{j}(Q) \partial_{y_{i}^{\prime}} \xi\left(y^{\prime}\right) d y^{\prime} \\
& +\int \frac{\left(x_{i}^{\prime}-y_{i}^{\prime}\right)\left(x^{\prime}-y^{\prime}\right)}{\left|x^{\prime}-y^{\prime}\right|^{4}} \cdot\left(2 m_{j}(Q)+m_{j}^{\prime}(Q) Q\right) \partial_{y^{\prime}} \xi\left(y^{\prime}\right) d y^{\prime} \\
& -\partial_{x_{i}^{\prime}} \eta(x) \int \frac{x^{\prime}-y^{\prime}}{\left|x^{\prime}-y^{\prime}\right|^{3}} \cdot m_{j}^{\prime}(Q) \partial_{y^{\prime}} \xi\left(y^{\prime}\right) d y^{\prime},
\end{aligned}
$$

which are of the form of the operators $C_{j}(\eta) \partial_{x^{\prime}} \xi$ defined in (4.40). The second and third terms of (4.59) consist of smoothing operators. The issue Vol. 14, $\mathrm{n}^{\circ}$ 5-1997. 
of the estimate is to have at most one derivative occur on each factor of $\eta\left(x^{\prime}\right)$. Calculating explicitly,

$$
\begin{aligned}
& \int \frac{1}{\left|x^{\prime}-y^{\prime}\right|^{2}+4 h^{2}} n_{j}{ }_{1}\left(Q_{1}, \frac{4 h}{\left(\left|x^{\prime}-y^{\prime}\right|^{2}+4 h^{2}\right)^{1 / 2}}\right) \partial_{y_{i}^{\prime}} Q_{1} \xi\left(y^{\prime}\right) d y^{\prime} \\
& -\int \frac{2\left(x_{i}^{\prime}-y_{i}^{\prime}\right)\left(x^{\prime}-y^{\prime}\right)}{\left(\left|x^{\prime}-y^{\prime}\right|^{2}+4 h^{2}\right)^{2}} \cdot n_{j-1}\left(Q_{1}, \frac{4 h}{\left(\left|x^{\prime}-y^{\prime}\right|^{2}+4 h^{2}\right)^{1 / 2}}\right) \partial_{y^{\prime}} Q_{1} \xi\left(y^{\prime}\right) d y^{\prime} \\
& +\int \frac{1}{\left|x^{\prime}-y^{\prime}\right|^{2}+4 h^{2}} \partial_{x^{\prime}}\left(n_{j-1}\left(Q_{1}, \frac{4 h}{\left(\left|x^{\prime}-y^{\prime}\right|^{2} \mid 4 h^{2}\right)^{1 / 2}}\right)\right) \partial_{y^{\prime}} Q_{1} \xi\left(y^{\prime}\right) d y^{\prime} \\
& +\int \frac{1}{\left|x^{\prime}-y^{\prime}\right|^{2}+4 h^{2}} n_{j-1}\left(Q_{1}, \frac{4 h}{\left(\left|x^{\prime}-y^{\prime}\right|^{2}+4 h^{2}\right)^{1 / 2}}\right) \partial_{y^{\prime}} \partial_{x_{i}^{\prime}} Q_{1} \xi\left(y^{\prime}\right) d y^{\prime} \\
& +\partial_{x_{i}^{\prime}} \int\left(\frac{4 h^{2} Q_{1}}{\left(\left|x^{\prime}-y^{\prime}\right|^{2}+4 h^{2}\right)^{2}} n_{j-1}\left(Q_{1}, \frac{4 h}{\left(\left|x^{\prime}-y^{\prime}\right|^{2}+4 h^{2}\right)^{1 / 2}}\right)\right. \\
& \left.+\frac{2 h}{\left(\left|x^{\prime}-y^{\prime}\right|^{2}+4 h^{2}\right)^{3 / 2}} n_{j}\left(Q_{1}, \frac{4 h}{\left(\left|x^{\prime}-y^{\prime}\right|^{2}+4 h^{2}\right)^{1 / 2}}\right)\right) \xi\left(y^{\prime}\right) d y^{\prime} .
\end{aligned}
$$

Terms which do not involve derivatives of $Q_{1}(\eta)$ are safe already. The terms which involve derivatives of $Q_{1}(\eta)$ give rise to the following four possible integrands;

$$
\begin{array}{cc}
\frac{Q_{1}^{j-1} \partial_{y^{\prime}} Q_{1}}{\left(\left|x^{\prime}-y^{\prime}\right|^{2}+4 h^{2}\right)^{r / 2}}, & \frac{Q_{1}^{j-1} \partial_{x_{i}^{\prime}} \partial_{y^{\prime}} Q_{1}}{\left(\left|x^{\prime}-y^{\prime}\right|^{2}+4 h^{2}\right)^{r / 2}} \\
\frac{Q_{1}^{j-2} \partial_{x_{i}^{\prime}} Q_{1} \partial_{y^{\prime}} Q_{1}}{\left(\left|x^{\prime}-y^{\prime}\right|^{2}+4 h^{2}\right)^{r / 2}}, & \frac{Q_{1}^{j-1} \partial_{x_{i}^{\prime}} Q_{1}}{\left(\left|x^{\prime}-y^{\prime}\right|^{2}+4 h^{2}\right)^{r / 2}}
\end{array}
$$

where

$$
\begin{aligned}
\partial_{y^{\prime}} Q_{1} & =\frac{\partial_{y^{\prime}} \eta\left(y^{\prime}\right)}{\left(\left|x^{\prime}-y^{\prime}\right|^{2}+4 h^{2}\right)^{1 / 2}}+\frac{\left(\eta\left(x^{\prime}\right)+\eta\left(y^{\prime}\right)\right)\left(x^{\prime}-y^{\prime}\right)}{\left(\left|x^{\prime}-y^{\prime}\right|^{2}+4 h^{2}\right)^{3 / 2}}, \\
\partial_{x_{i}^{\prime}} Q_{1} & =\frac{\partial_{x^{\prime}} \eta\left(x^{\prime}\right)}{\left(\left|x^{\prime}-y^{\prime}\right|^{2}+4 h^{2}\right)^{1 / 2}}-\frac{\left(\eta\left(x^{\prime}\right)+\eta\left(y^{\prime}\right)\right)\left(x_{i}^{\prime}-y_{i}^{\prime}\right)}{\left(\left|x^{\prime}-y^{\prime}\right|^{2}+4 h^{2}\right)^{3 / 2}}, \\
\partial_{y^{\prime}} \partial_{x_{i}^{\prime}} Q_{1} & =-\frac{\partial_{y} \eta\left(y^{\prime}\right)\left(x_{i}^{\prime}-y_{i}^{\prime}\right)}{\left(\left|x^{\prime}-y^{\prime}\right|^{2}+4 h^{2}\right)^{3 / 2}} \\
& -3 \frac{\left(\eta\left(x^{\prime}\right)+\eta\left(y^{\prime}\right)\right)\left(x^{\prime}-y^{\prime}\right)\left(x_{i}^{\prime}-y_{i}^{\prime}\right)}{\left(\left|x^{\prime}-y^{\prime}\right|^{2}+4 h^{2}\right)^{5 / 2}} \\
& +\frac{\partial_{x_{i}^{\prime}} \eta\left(x^{\prime}\right)\left(x^{\prime}-y^{\prime}\right)+\left(\eta\left(x^{\prime}\right)+\eta\left(y^{\prime}\right)\right) \mathbf{e}_{\mathbf{i}}}{\left(\left|x^{\prime}-y^{\prime}\right|^{2}+4 h^{2}\right)^{3 / 2}}
\end{aligned}
$$

where $\mathbf{e}_{\mathbf{i}}$ is the $i^{\text {th }}$ unit vector. These smoothing operators are of the form $C_{p, h}(\eta) \xi$ studied in Section 5 , that have already appeared in the analysis of $B_{j}$. 
The operators $A_{J}^{R}$ resulting from the Taylor series remainders of the expansion of $A$ behave similarly. These kernels contain functions of $Q$ and $Q_{1}$ which are analytic in their arguments and the results of Section 5 are designed for this situation.

We conclude this section by stating the result that gives an estimate of the remainders $R_{1}, R_{2}$ and $R_{3}(\eta)$, which are defined in (4.26) and (4.27), from the Taylor expansion of $G(\eta)$ to the first several orders.

Theorem 4.7. - Consider $1<q<+\infty$ and $0 \leq s \in \mathbf{N}$. Let $|\eta|_{C^{1}}<R_{0}$ and $|\eta|_{C^{s+1}}<+\infty$ The Taylor remainders $R_{j}(\eta)$ from the expansion of $G(\eta) j=1,2,3$ satisfy

$$
\left\|R_{j}(\eta) \xi\right\|_{s, q} \leq C(s)|\eta|_{C^{1}}^{j-1}\left(|\eta|_{C^{1}}\|\xi\|_{s+1, q}+|\eta|_{C^{s+1}}\|\xi\|_{1, q}\right)
$$

\section{ESTIMATES OF SINGULAR INTEGRALS}

This section is focused on the analysis of the singular integral operators that are described in Section 4, and which make up the components of the Dirichlet-Neumann operator. Although the majority of this paper concerns two-dimensional surfaces of fluid regions in three dimensions, here we will give general results for $n$-dimensional singular integrals. The two basic operators are

$$
C_{p}(\eta) \xi(x)=\int_{\mathbf{R}^{n}} k(x-y) c_{p}(Q(\eta)) \xi(y) d y
$$

where $k(x)$ is a convolution kernel of Calderon-Zygmund class satisfying the so-called standard estimates for $\delta>0$ :

$$
\begin{gathered}
|k(x)| \leq \frac{C_{1}}{|x|^{n}}, \quad \forall x \in \mathbf{R}^{n} \\
|k(x)-k(y)| \leq \frac{C_{1}}{|x|^{n+\delta}}|x-y|^{\delta}, \\
\forall x, y \in \mathbf{R}^{n}, \quad \text { with }|x-y| \leq \frac{1}{2}|x| .
\end{gathered}
$$

The expression $Q(\eta)=(\eta(x)-\eta(y)) /|x-y|$ is the ubiquitous difference quotient. The kernel is specified by the function $c_{p}(z)$, which is assumed to be analytic in the ball $|z|<R_{0}$ and to satisfy $c_{p}(z) \sim O\left(|z|^{p}\right)$, and we might as well take it to the real for $z$ real. Notice that $|Q(\eta)|_{L^{\infty}} \leq\left|\partial_{x} \eta\right|_{L^{\infty}}$, the Lipschitz norm of $\eta(x)$, so that for $\left|\partial_{x} \eta\right|_{L^{\infty}}<R_{0}$ the kernel is well defined. 
In addition to operators of type (5.1) it is necessary to study related smoothing operators, which were introduced through the Neumann boundary conditions on the bottom of the fluid region. These have the form

$$
\begin{aligned}
& C_{p, h}(\eta) \xi(x) \\
& \quad=\int_{\mathbf{R}^{n}} k_{h}(x-y) c_{p, h}\left(Q_{1}(\eta), \frac{4 h}{\left(|x-y|^{2}+4 h^{2}\right)^{1 / 2}}\right) \xi(y) d y
\end{aligned}
$$

where $Q_{1}(\eta)=(\eta(x)+\eta(y)) /\left(|x-y|^{2}+4 h^{2}\right)^{1 / 2}$ and the function $c_{p, h}(z, w)$ is analytic over the set $|z|<R_{0},|w| \leq 2$, with $\left|c_{p, h}(z, w)\right| \sim O\left(|z|^{p}|w|^{r}\right)$. The convolution kernel is a smoothing kernel of the form

$$
k_{h}(x-y)=\frac{1}{\left(|x-y|^{2}+4 h^{2}\right)^{\rho / 2}} \prod_{\ell=1}^{n}\left(\frac{(x-y)_{\ell}}{\left(|x-y|^{2}+4 h^{2}\right)^{1 / 2}}\right)^{n_{\ell}} .
$$

Again notice that for $\left|Q_{1}(\eta)\right|_{L^{\infty}} \leq|\eta|_{L^{\infty}} / h<R_{0}$, the integrand of (5.3) is well defined. We will prove in this section the following two principal results.

THEOREM 5.1. - For $|\eta|_{C^{1}}<R_{0}$ and $|\eta|_{C^{s+1}}<+\infty$ then $C_{p}(\eta)$ is a bounded operator on $W^{s, q}\left(\mathbf{R}^{n}\right)$, and

$$
\left\|C_{p}(\eta) \xi\right\|_{s, q} \leq|\eta|_{C^{1}}^{p-1}\left(|\eta|_{C^{1}}\left\|\partial_{x}^{s} \xi\right\|_{q}+C(s)|\eta|_{C^{s+1}}\|\xi\|_{q}\right) .
$$

Furthermore, the operator $C_{p}(\xi)$ is analytic as a mapping on $W^{s, q}\left(\mathbf{R}^{n}\right)$, for $\eta$ in the set

$$
\left\{\eta:|\eta|_{C^{1}}<R_{0},\left|\partial_{x} \eta\right|_{C^{s}}<+\infty\right\} \subseteq C^{s+1}\left(\mathbf{R}^{n}\right)
$$

and thus its Taylor series representation is convergent in operator norm.

Theorem 5.2. - Let $p+r+\rho>n$, and suppose that $|\eta|_{L^{\infty}}<h R_{0}$, $|\eta|_{C s}<+\infty$. Then

$$
\left\|C_{p, h}(\eta) \xi\right\|_{s, q}<C(s)|\eta|_{L^{\infty}}^{p-1}|\eta|_{C^{s}}|| \xi \|_{q}
$$

Furthermore the operator $C_{p, h}(\eta)$ is analytic as a mapping of $W^{s, q}$ to $L^{q}$, for $\eta(x)$ in the set $\left\{\eta:|\eta|_{L^{\infty}}<h R_{0},|\eta|_{C^{\circ}}<+\infty\right\}$, and is thus represented by its Taylor series approximation.

The proofs of these two results use an $L^{q}$ estimate on operators of type (5.1), combined with control on the order $s$ of the Sobolev space in question, with attention to the growth of the constants in both $p$ and $s$. 
Clearly the more sensitive of the two theorems is Theorem 5.1. The form of result that we need is the following.

THEOREM 5.3. - Consider functions $\eta_{1}(x), \ldots, \eta_{p}(x) \in C^{1}\left(\mathbf{R}^{n}\right)$ and $k(x)$ a singular integral kernel satisfying the standard estimates (5.2). Then the homogeneous operator of degree $p$,

$$
S_{p}\left(\eta_{1}, \ldots, \eta_{p}\right) \xi(x)=\int k(x-y) \prod_{j=1}^{p} Q\left(\eta_{j}\right) \xi(y) d y
$$

is bounded on $L^{q}\left(\mathbf{R}^{n}\right)$, and satisfies the estimate

$$
\left\|S_{p}\left(\eta_{1}, \ldots, \eta_{p}\right) \xi\right\|_{q} \leq C_{0} p^{M} \prod_{j=1}^{p}\left|\eta_{j}\right|_{C^{1}}\|\xi\|_{q}
$$

with exponent $M=3+\delta$.

The proof is fundamentally based on the following theorem of M. Christ and J.L. Journé, [3] on $L^{q}$ bounds for Calderon Zygmund commutators.

THEOREM 5.4 ([3], Theorem 4), - Consider the singular integral operator $L$ with kernel

$$
L(x, y)=K(x-y) \prod_{j=1}^{p}\left(\int_{0}^{1} b_{j}(t x+(1-t) y) d t\right)
$$

where each $b_{j} \in L^{\infty}\left(\mathbf{R}^{n}\right)$, and $K(x)$ satisfies the standard estimates (5.2). Then

$$
\left\|\int L(x, y) \xi(y) d y\right\|_{q} \leq C(\delta, q) C_{1} p^{N}\left(\prod_{j=1}^{p}\left|b_{j}\right|_{L^{\infty}}\right)\|\xi\|_{q} .
$$

In fact one may take $N=2+\delta$, where $\delta$ appears in the standard estimates (5.2), as does the constant $C_{1}$.

Proof of Theorem 5.3. - The integral kernel is

$$
\begin{gathered}
k(x-y) \prod_{j=1}^{p} Q\left(\eta_{j}\right)=k(x-y) \sum_{\text {permutations }} \prod_{\ell_{i}}^{p} \frac{x_{\ell_{i}}-y_{\ell_{i}}}{|x-y|} \\
\times \int_{0}^{1} \partial_{x_{\ell_{i}}} \eta_{j}(t x+(1-t) y) d t
\end{gathered}
$$


since

$$
Q\left(\eta_{j}\right)=\sum_{i=1}^{n} \frac{x_{\ell}-y_{\ell}}{|x-y|} \int_{0}^{1} \partial_{x_{\ell}} \eta_{j}(t x+(1-t) y) d t .
$$

The sum is taken over all possible $\ell_{1}, \ell_{2}, \ldots, \ell_{p}$ betwcen 1 and $n$ (giving $n^{p}$ many terms). For each summand the result is an integral operator with a convolution kernel of the form

$$
K(x)=k(x) \prod_{i=1}^{p} \frac{x_{\ell_{i}}}{|x|}
$$

and whose remaining factor is

$$
\prod_{j=1}^{p} \int_{0}^{1} b_{\ell_{j} j}(t x+(1-t) y) d t
$$

with $b_{m j}(x)=\partial_{x_{m}} \eta_{j}(x)$. Such operators are the subject of the paper of Christ and Journé, which gives the $L^{q}\left(\mathbf{R}^{n}\right)$ bounds.

We would like to remark that we are using $K(x)=k(x) \Pi_{i=1}^{p} x_{\ell_{i}} /|x|$, so that it itself depends upon the power $p$, and the constant $C_{1}$ in the standard estimate (5.2) will be affected. This changes the ultimate power of $p$ that appears in Theorem 5.3.

LEMma 5.5. - If $k(x)$ satisfies the standard estimate (5.2) with constants $C_{1}$ and $0<\delta \leq 1$, then the kernels

$$
K(x)=k(x) \prod_{i=1}^{p} \frac{x_{\ell_{i}}}{|x|}
$$

satisfy the standard estimates, with constants $C(p)=O\left(p C_{1}\right)$.

Proof. - Clearly the product does not change (5.2)(i), for we have

$$
|K(x)|=|k(x)| \prod_{i=1}^{p} \frac{\left|x_{\ell_{i}}\right|}{|x|} \leq \frac{C_{1}}{|x|^{n}} .
$$

The estimate (5.2)(ii) is

$$
\begin{aligned}
|K(x)-K(y)| & \leq|k(x)-k(y)| \prod_{\ell=1}^{n} \frac{\left|x_{\ell}\right|}{|x|}+|k(y)| \sum_{j=1}^{p}\left|\frac{x_{\ell_{j}}}{|x|}-\frac{y_{\ell_{j}}}{|y|}\right| \\
& \leq 3 p \frac{C_{1}}{|x|^{n+\delta}}|x-y|^{\delta}
\end{aligned}
$$

for $|x-y| \leq \frac{1}{2}|x|$ 
Using this lemma and Theorem 5.4, we have essentially completed the proof of Theorem 5.3. Each of the terms in (5.11) is estimated in the $L^{q}$ bound of (5.9),

$$
\left\|S_{p}\left(\eta_{1}, \cdots \eta_{p}\right) \xi\right\|_{q} \leq C(p) p^{N}\left(\sum_{\text {permutations }} \prod_{\ell_{i}}^{p}\left|\partial_{j=1} \eta_{x_{l_{i}}} \eta_{j}\right|_{L \infty}\right)\|\xi\|_{q}
$$

Since

$$
\sum_{\text {permutations }} \prod_{\ell_{i}}^{p}\left|\partial_{x_{l_{i}}} \eta_{j}\right|_{L^{\infty}} \leq \prod_{j=1}^{p}\left|\eta_{j}\right|_{C^{1}}
$$

and the constant $C(p)=p C_{1}$ is linear in $p$, we have the estimate

$$
\left\|S_{p}\left(\eta_{1}, \cdots \eta_{p}\right) \xi\right\|_{q} \leq C_{0} p^{M}\left(\prod_{j=1}^{p}\left|\partial_{x} \eta_{j}\right|_{L^{\infty}}\right)\|\xi\|_{q}
$$

with $M=N+1=3+\delta$.

To continue the section we move to estimates of these singular integral operators in higher Sobolev norms. For this we use the following lemma on the commutation of differentiation with singular integral operators $S_{p}\left(\eta_{1}, \cdots \eta_{p}\right)$ defined in (5.7).

LEMMA 5.6. - Assume that $k(x)$ is smooth away from $x=0$, and that $c_{p}\left(Q_{1}, \cdots, Q_{p}\right)$ is a smooth function of the difference quotients $Q_{j}=Q\left(\eta_{j}\right)$. Then

$$
\begin{aligned}
\partial_{x}( & \left.(x-y) c_{p}\left(Q_{1}, \cdots Q_{p}\right)\right) \\
\quad= & -\partial_{y}\left(k(x-y) c_{p}\left(Q_{1}, \cdots Q_{p}\right)\right) \\
& \quad+k(x-y) \sum_{j=1}^{p} \partial_{Q_{j}} c_{p}\left(Q_{1}, \cdots Q_{p}\right) Q\left(\partial_{x} \eta_{j}\right)
\end{aligned}
$$

Proof. - We simply check the calculation that

$$
\begin{gathered}
\partial_{x} Q\left(\eta_{j}\right)=\frac{\partial_{x} \eta_{j}(x)}{|x-y|}-\frac{\eta_{j}(x)-\eta_{j}(y)}{|x-y|^{3}}(x-y) \\
\partial_{y} Q\left(\eta_{j}\right)=-\frac{\partial_{x} \eta_{j}(y)}{|x-y|}+\frac{\eta_{j}(x)-\eta_{j}(y)}{|x-y|^{3}}(x-y),
\end{gathered}
$$

thus $\partial_{x} Q\left(\eta_{j}\right)+\partial_{y} Q\left(\eta_{j}\right)=Q\left(\partial_{x} \eta_{j}\right)$. 
From Lemma 5.6 the commutator of operators (5.1) or (5.7) with partial derivatives is given by the expression

$$
\partial_{x} S_{p}\left(\eta_{1}, \cdots, \eta_{p}\right) \xi(x)=S_{p}\left(\eta_{1}, \cdots, \eta_{p}\right) \partial_{x} \xi+S_{p}^{\prime} \xi
$$

where $S_{p}^{\prime}$ is of the same form as (5.7), with integrand

$$
k(x-y) \sum_{j=1}^{p}\left(\prod_{\substack{\ell=1 \\ \ell \neq j}}^{p} Q\left(\eta_{\ell}\right)\right) Q\left(\partial_{x} \eta_{j}\right)
$$

Using Lemma 5.6 recursively, we have that

$$
\partial_{x}^{s} S_{p}(\eta, \cdots, \eta) \xi(x)=\sum_{0 \leq|\tau| \leq s} \sum_{\substack{\alpha \in \mathbb{N}^{p} \\|\alpha+\tau|=s}} S_{p}\left(\partial_{x}^{\alpha_{1}} \eta, \cdots \partial_{x}^{\alpha_{p}} \eta\right) \partial_{x}^{\tau} \xi
$$

Notice that there are $p^{s-|\tau|}$-many terms in the summand, in the case that $|\tau|$-many derivatives fall on the function $\xi(x)$. From Theorem 5.3 we can bound the resulting operator;

$$
\begin{aligned}
\left\|\partial_{x}^{s} S_{p}(\eta, \cdots \eta) \xi\right\|_{q} & \leq \sum_{|\alpha+\tau|=s}\left\|S_{p}\left(\partial_{x}^{\alpha_{1}} \eta, \cdots \partial_{x}^{\alpha_{p}} \eta\right) \partial_{x}^{\tau} \xi\right\|_{q} \\
& \leq \sum_{|\alpha+\tau|==} C_{0} p^{M}\left(\prod_{j=1}^{p}\left|\partial_{x}^{\alpha_{j}} \eta\right|_{C^{1}}\right)\left\|\partial_{x}^{\tau} \xi\right\|_{L^{4}}
\end{aligned}
$$

Standard interpolation gives that $\left|\partial_{x}^{\alpha} \eta\right|_{C^{1}} \leq|\eta|_{C^{1}}^{1-\alpha / m}\left|\partial_{x}^{m} \eta\right|_{C^{1}}^{\alpha / m}$, thus our operator estimate becomes

$$
\begin{aligned}
& \left\|\partial_{x}^{s} S_{p}(\eta, \cdots, \eta) \xi\right\|_{q} \leq \sum_{|\alpha+\tau|=s} C_{0} p^{M}\left(\prod_{j=1}^{p}\left|\partial_{x}^{\alpha_{j}} \eta\right|_{C^{1}}\right)\left\|\partial_{x}^{\tau} \xi\right\|_{q} \\
& \quad \leq \sum_{|\alpha+\tau|=s} C_{0} p^{M} \prod_{j=1}^{p}\left(|\eta|_{C^{1}}^{1-\left|\alpha_{j}\right| / m}\left|\partial_{x}^{m} \eta\right|_{C^{1}}^{\left|\alpha_{j}\right| / m}\right)\left\|\partial_{x}^{\tau} \xi\right\|_{q} \\
& \quad \leq \sum_{|\alpha+\tau|=s} C_{0} p^{M}|\eta|_{C^{1}}^{p-1}\left|\partial_{x}^{m} \eta\right|_{C^{1}}\left\|\partial_{x}^{\tau} \xi\right\|_{q} \quad \text { for } m=s-|\tau| .
\end{aligned}
$$

Using the second interpolation that $\left|\partial_{x}^{m} \eta\right|_{C^{1}}\left\|\partial_{x}^{\tau} \xi\right\|_{q} \leq\left(|\eta|_{C^{\perp}}\left\|\partial_{x}^{s} \xi\right\|_{q}+\right.$ $\left.C(s)\left|\partial_{x}^{s} \eta\right|_{C^{1}}\|\xi\|_{q}\right)$, for $|\tau|+m=s$, we have shown that the $L^{q}$-norm of 
(5.17) is bounded, in fact we have proved the following, since there are at most $p^{s}$-many terms on the r.h.s. of (5.19).

Theorem 5.7. - Suppose that $1<q<+\infty$, and that $\eta \in C^{s+1}\left(\mathbf{R}^{n}\right)$, and consider the mapping properties of the operator $S_{p}(\eta, \cdots, \eta)$. We have the estimate

$$
\begin{aligned}
& \left\|\partial_{x}^{s} S_{p}(\eta, \cdots, \eta) \xi\right\|_{q} \\
& \quad \leq p^{M+s}|\eta|_{C^{1}}^{p-1}\left(|\eta|_{C^{1}}\left\|\partial_{x}^{s} \xi\right\|_{q}+C(s)|\eta|_{C^{s+1}}\|\xi\|_{q}\right)
\end{aligned}
$$

Proofs of Theorems 5.1 and 5.2. - The aim is to address the general case of an integral kernel which is a general analytic function of the quotient $Q(\eta)$, in the form of the operators (5.1). Using Taylor series to describe $c_{p}(z)=\sum_{m \geq p}\left(c_{m} / m !\right) z^{m}$ gives us the definition

$$
C_{p}(\eta) \xi(x)=\sum_{m \geq p} \frac{c_{m}}{m !} S_{m}(\eta, \cdots, \eta) \xi(x) .
$$

Considered as an operator on $L^{q}\left(\mathbf{R}^{n}\right)$, this series converges in operator norm for $|\eta|_{C^{1}} \leq R<R_{0}$. Indeed, because of estimate (5.8), the Taylor coefficients of $C_{p}(\eta)$ grow no faster than $C_{0}\left|c_{m}\right| m^{M}|\eta|_{C^{1}}^{m}$, which is bounded by some $C m ! R^{m}$ by comparison with the Taylor series for the function $z^{M} \partial_{z}^{M} c_{p}(z)$ which is also analytic on the ball $|z|<R_{0}$, of course.

This behavior is not drastically modified when considering $C_{p}(\eta)$ on $W^{s, q}\left(\mathbf{R}^{n}\right)$; we work with the estimates of Theorem 5.7 to obtain

$$
\begin{aligned}
& \left\|\partial_{x}^{s} \sum_{m \geq p} \frac{c_{m}}{m !} S_{m}(\eta, \cdots, \eta) \xi(x)\right\|_{q} \\
& \quad \leq \sum_{m \geq p} \frac{\left|c_{m}\right|}{m !} m^{M+s}|\eta|_{C^{1}}^{m-1}\left(|\eta|_{C^{1}}\left\|\partial_{x}^{s} \xi\right\|_{q}+C(s)|\eta|_{C^{s+1}}\|\xi\|_{q}\right)
\end{aligned}
$$

This is again seen to be convergent in operator norm, as the Taylor coefficients are again controlled by $C m ! R^{m}$ when $|\eta|_{C^{1}} \leq R<R_{0}$, by comparison with the Taylor series of the analytic function $z^{M+s} \partial_{z}^{M+s} c_{p}(z)$ which has the same radius of convergence as $c_{p}$. When we recognize that $r_{p}(z) \sim O\left(|z|^{p}\right)$ this gives the result stated in Theorem 5.1.

The remaining subject of this section is the proof of Theorem 5.2, concerning the smoothing operators $C_{p, h}(\eta)$. These are of the form (5.3), with smoothing kernels described in (5.4). The number of factors $\sum_{\ell=1}^{n} r_{\ell}$ 
in the product (5.4) need not grow in $p$. Using the analyticity of $c_{p}(z, w)$ we can represent the smoothing operators in Taylor series,

$$
\begin{aligned}
C_{p, h}(\eta) \xi(x)= & \sum_{j, \ell} \frac{\left(c_{p, h}\right)_{j, \ell}}{j ! \ell !} \\
& \times \int k_{h}(x-y)\left(\frac{4 h}{\left(|x-y|^{2}+4 h^{2}\right)^{1 / 2}}\right)^{\ell} Q_{1}^{j}(\eta) \xi(y) d y
\end{aligned}
$$

The conclusion of Theorem 5.2 will follow from an estimate in $L^{q}\left(\mathbf{R}^{n}\right)$ of the smoothing operators

$$
S_{p, h}(\eta) \xi(x)=\int k_{h}(x-y)\left(\frac{4 h}{\left(|x-y|^{2}+4 h^{2}\right)^{1 / 2}}\right)^{\ell} Q_{1}^{p}(\eta) \xi(y) d y,
$$

and their derivatives. In order that the integrand be absolutely integrable, we ask that $\rho+\ell+p>n$.

THEOREM 5.8. - In case $\rho+\ell+p>n$ and both $|\eta|_{L^{\infty}}<h R_{0}$, $\left|\partial_{x}^{s} \eta\right|_{L^{\infty}}<+\infty$, then the estimates hold;

$$
\begin{aligned}
& \left\|S_{p, h}(\eta) \xi\right\|_{q} \leq C_{0}\left(|\eta|_{L^{\infty}} / h\right)^{p}\|\xi\|_{q} \\
& \left\|\partial_{x}^{s} S_{p, h}(\eta) \xi\right\|_{q} \leq \frac{C_{0}}{h}(p)^{s}\left(|\eta|_{L^{\infty}} / h\right)^{p-1}|\eta|_{C^{s}}\|\xi\|_{q}
\end{aligned}
$$

Using this estimate in a manner similar to the proof of Theorem 5.1, the results of Theorem 5.2 will also follow.

Proof. - The simple $L^{q}$-bounds on $S_{p, h}(\eta)$ come from the expression

$$
\left\|S_{p, h}(\eta) \xi\right\|_{q}<\sup _{x \in \mathbf{R}^{n}} \int\left|k_{h}(x-y)\left(\frac{4 h}{\left(|x-y|^{2}+4 h^{2}\right)^{1 / 2}}\right)^{\ell} Q_{1}^{p}(\eta)\right| d y\|\xi\|_{q}
$$

Since

$$
Q_{1}(\eta)=\frac{2 h}{\left(|x-y|^{2}+4 h^{2}\right)^{1 / 2}} \frac{\eta(x)+\eta(y)}{2 h}
$$

then

$$
\left\|S_{p, h}(\eta) \xi\right\|_{q} \leq \int\left(\frac{4 h}{\left(|y|^{2}+4 h^{2}\right)^{1 / 2}}\right)^{\rho+\ell+p} d y\left(|\eta|_{L^{\infty}} / h\right)^{p}\|\xi\|_{q}
$$


which is what is needed. The derivative estimate follows from the Leibnitz rule;

$$
\begin{aligned}
\partial_{x}^{s} S_{p, h}(\eta) \xi(x)= & \sum_{\substack{m=0\\
}} \int \partial_{x}^{s-m}\left(k_{h}(x-y) \frac{(4 h)^{\ell}}{\left(|x-y|^{2}+4 h^{2}\right)^{\ell / 2}}\right) \\
& \times \partial_{x}^{m}\left(Q_{1}^{p}(\eta)\right) \xi(y) d y .
\end{aligned}
$$

This is of course similar to (5.26), once we count the number of derivatives which have fallen on $\eta(x)$. This is bounded by

$$
\begin{aligned}
\left|\partial_{x}^{m} Q_{1}^{p}(\eta)\right| & =\left|\sum_{\tau=0}^{m} \partial_{x}^{\tau}(\eta(x)+\eta(y))^{p} \partial_{x}^{m-\tau}\left(\frac{1}{\left(|x-y|^{2}+4 h^{2}\right)^{1 / 2}}\right)^{p}\right| \\
& \leq \frac{C}{h} p^{m}\left(|\eta|_{L^{\infty}} / h\right)^{p-1}|\eta|_{C^{s}} .
\end{aligned}
$$

There are s-many terms in the sum (5.27) as well. Counting them all, we have finished the proof of Theorem 5.8, and thus also Theorem 5.2, ending the section on Sobolev bounds for the relevant integral operators.

\section{JUSTIFICATION OF THE MODULATION APPROXIMATION}

\subsection{Preliminaries}

We denote by $\mathbf{W}(\eta, \xi)$ the water wave operator defined by

$$
\mathbf{W}(\eta, \xi)=\left(\begin{array}{l}
W_{1}(\eta, \xi) \\
W_{2}(\eta, \xi)
\end{array}\right)
$$

where $W_{1}$ and $W_{2}$ are defined as the 1.h.s of equations (2.7) and respectively (2.8);

$$
\begin{aligned}
W_{1}= & \partial_{t} \eta-G(\eta) \xi \\
W_{2}= & \partial_{t} \xi+g \eta+\frac{1}{2\left(1+\left|\partial_{x^{\prime}} \eta\right|^{2}\right)}\left(\left|\partial_{x^{\prime}} \xi\right|^{2}-(G(\eta) \xi)^{2}\right. \\
& \left.-2\left(\partial_{x^{\prime}} \eta \cdot \partial_{x^{\prime}} \xi\right) G(\eta) \xi+\left|\partial_{x^{\prime}} \eta\right|^{2}\left|\partial_{x^{\prime}} \xi\right|^{2}-\left(\partial_{x^{\prime}} \eta \cdot \partial_{x^{\prime}} \xi\right)^{2}\right)-\beta H(\eta)
\end{aligned}
$$

A solution of the water wave problem satisfies $\mathbf{W}(\eta, \xi)-0$. In fact this is an initial value problem for the functions $\left(\eta\left(x^{\prime}, t\right), \xi\left(x^{\prime}, t\right)\right)$.

In Section 3 we have constructed a formal approximation of the solution of the water-wave problem (2.7)-(2.8), based on a description of the modulational regime given through the functions $c\left(z_{1}, z_{2}, \tau\right)$ and $d\left(z_{1}, z_{2}, \tau\right)$ Vol. 14, n 5-1997. 
which satisfy the Davey-Stewartson system (3.24). In the physical variables given by $z_{1}=\epsilon\left(x_{1}-\omega^{\prime} t\right), z_{2}=\epsilon x_{2}$, and $\tau=\epsilon^{2} t$, the approximate solution is given by

$$
\begin{aligned}
& \tilde{\eta}=\epsilon \eta^{(1)}+\epsilon^{2} \eta^{(2)}+\epsilon^{3} \eta^{(3)} \\
& \tilde{\xi}=\epsilon \xi^{(1)}+\epsilon^{2} \xi^{(2)}+\epsilon^{3} \xi^{(3)}
\end{aligned}
$$

where $\left(\eta^{(1)}, \xi^{(1)}\right)$ is defined by the expressions

$$
\begin{aligned}
& \eta^{(1)}=\frac{i \omega}{g+\beta k_{1}^{2}} c e^{i \varphi}+c . c \\
& \xi^{(1)}=c e^{i \varphi}+c \cdot c+d .
\end{aligned}
$$

The formal procedure also gives the higher order components of this expression, $\left(\eta^{(2)}, \xi^{(2)}\right)$ and $\left(\eta^{(3)}, \xi^{(3)}\right)$, which are respectively (see (3.19) and (3.25))

$$
\begin{aligned}
& \eta^{(2)}=\alpha_{1} c_{z_{1}} e^{i \varphi}+\alpha_{2} c^{2} e^{2 i \varphi}+c . c+\alpha_{3}|c|^{2}+\alpha_{4} d_{z_{1}} \\
& \xi^{(2)}=\alpha_{2}^{\prime} c^{2} e^{2 i \varphi}+c . c \\
\eta^{(3)}= & \delta_{1} c^{3} e^{3 i \varphi}+\delta_{2} c c_{z_{1}} e^{2 i \varphi} \\
& +\left(\delta_{3} c_{z_{1} z_{1}}+\delta_{4} c_{z_{2} z_{2}}+\delta_{5}|c|^{2} c+\delta_{6} c d_{z_{1}}\right) e^{i \varphi}+c \cdot c+\delta_{7} \\
\xi^{(3)}= & \delta_{1}^{\prime} c^{3} e^{3 i \varphi}+\delta_{2}^{\prime} c c_{z_{1}} e^{2 i \varphi}+c \cdot c,
\end{aligned}
$$

with coefficients $\alpha_{i}, \alpha_{i}^{\prime}, \delta_{j}, \delta_{j}^{\prime}$ depending on $\omega, k, h, \beta$ and $g$ and $\varphi=k_{1} x_{1}-\omega t$.

The basic question that we are to address in this article is the extent to which the above formal solution deviates from being a true solution of the full Euler equation description of the evolution of the free surface, given by a solution of the system $\mathbf{W}(\eta, \xi)=0$. More precisely, consider the initial condition $\left(\eta_{0}, \xi_{0}\right)=\left.(\widetilde{\eta}, \widetilde{\xi})\right|_{t=0}$, and let $(\eta, \xi)$ be the solution of the initial value problem $\mathbf{W}(\eta, \xi)=0$ with the initial condition $\left(\eta_{0}, \xi_{0}\right)$. The strongest possible result would be to compare the exact solution $(\eta, \xi)$ of $(6.1)$ with the modulation approximation $(\tilde{\eta}, \widetilde{\xi})$ in an appropriate norm, with an estimate of the form

$$
\sup _{t \in I}\|(\widetilde{\eta}, \widetilde{\xi})-(\eta, \xi)\| \leq o\left(\epsilon^{3}\right)
$$


over an interval of time $I=\left[0, t_{0}\right]$ with $t_{0}=O\left(\epsilon^{-2}\right)$. Such a result would require an existence theory for the water wave in three space dimensions for solutions which do not develop singularities over long time intervals, given initial conditions of size $O(\epsilon)$. This is currently an open mathematically problem, whose resolution is not given in this paper. We limit ourselves instead to presenting a weaker result, evaluating the accuracy to which the modulational solution $(\widetilde{\eta}, \widetilde{\xi})$ satisfies the water wave problem. Equivalently, we give an estimate of $\mathbf{W}(\widetilde{\eta}, \widetilde{\xi})$ in a Sobolev norm, and show that it is $o\left(\epsilon^{3}\right)$ uniformly in the time interval $I$. This is already a challenge, due to the singular nature of the formal derivation, in which integral operators of potential theory (with coefficients depending on multiple scales) are approximated by differential operators, and error terms contain high derivatives of the basic dependent variables.

Before we proceed with the analysis, we need to introduce some tools concerning the mathematical theory of the Davey-Stewartson system and the analysis of pseudo-differential operators with their action on multiplescale functions. This is the object of the two following sections. Finally, in the last section (Section 6.4), we establish an estimate for $\mathbf{W}(\widetilde{\eta}, \widetilde{\xi})$.

\subsection{The Davey-Stewartson system}

In this paragraph, we recall some mathematical results for the initial value problem for the Davey-Stewartson system (3.24). After simple rescaling, one can reduce the system to

$$
\begin{aligned}
& i c_{\tau}+\delta c_{z_{z_{1} z_{1}}}+c_{z_{2} z_{2}}=\lambda|c|^{2} c+b c d_{z_{1}} \\
& d_{z_{1} z_{1}}+m d_{z_{2} z_{2}}=|c|_{z_{1}}^{2}
\end{aligned}
$$

with the initial condition $c\left(z_{1}, z_{2}, 0\right)=c_{0}\left(z_{1}, z_{2}\right)$. The coefficients $\delta$ and $\lambda$ can be normalized to be of absolute value 1 . The character of the solutions depends strongly on the sign of the parameters $\delta$ and $m$. Indeed, the system is classified as elliptic-elliptic, elliptic-hyperbolic, hyperbolicelliptic and hyperbolic-hyperbolic according to the respective signs of $\delta$ and $m$. However, the hyperbolic-hyperbolic case is not encountered in physical situations.

Boundary conditions depend on the sign of $m$ as discussed in [1]. For $m>0$, they reduce to $c \rightarrow 0$ and $d \rightarrow 0$ when $z_{1}^{2}+z_{2}^{2} \rightarrow \infty$. For $m<0$, they are of radiation type, that is, $c \rightarrow 0$ when $z_{1}^{2}+z_{2}^{2} \rightarrow \infty$ and $d \rightarrow 0$ when the characteristic variables $z_{1} \pm \sqrt{-m} z_{2} \rightarrow+\infty$, with no condition when $z_{1} \pm \sqrt{-m} z_{2} \rightarrow-\infty$.

We report below the main results concerning strong solutions. Detailed study of strong and weak solutions, together with long time behavior can be found in [13], [14], [16] and [20] and [26]. 
THEOREM 6.1. - Suppose $m>0$. Given an initial condition $c_{0}$ in $H^{r}\left(\mathbf{R}^{2}\right)$, there exists a unique solution $(c, d)$ during a finite interval of time $\left[0, \tau_{1}\right)$ such that $c \in C\left(\left[0, \tau_{1}\right), H^{r}\left(\mathbf{R}^{2}\right)\right)$ and $d \in C\left(\left[0, \tau_{1}\right), H^{r+1}\left(\mathbf{R}^{2}\right)\right)$.

In the elliptic-elliptic case, the situation is similar to that of the cubic Schrödinger equation: if $\lambda \geq \max (-b, 0)$, smooth solutions can be extended for all time, while if $\lambda<\max (-b, 0)$, solutions may focus in a finite time [13]. The structure of the singular solutions is critical as in the twodimensional cubic Schrödinger equation [24]. In the hyperbolic-elliptic case, smooth solutions can be extended for all time if the $L^{2}$-norm of the initial conditions is small enough [13].

The elliptic-hyperbolic and hyperbolic-hyperbolic cases are more complex. Existence of smooth solutions has been proved first by Linares and Ponce for small initial data in weighted Sobolev spaces [20]. In a recent work, Hayashi and Saut [16] proved local existence of sulutions in some analytic function spaces without a smallness condition on data. They also proved global existence of small solutions. We state below a recent result by Guzmán-Gómez [14] in usual Sobolev spaces that gives a bound on the $L^{2}$-norm of the initial condition to ensure local existence.

Theorem 6.2. - Suppose $\delta>0$ and $m<0$. For initial condition $c_{0} \in H^{3}\left(\mathbf{R}^{2}\right)$ satisfying $\left|c_{0}\right|_{L^{2}}^{2}<\frac{\sqrt{-m}}{2|b|}$, there exists a unique solution $(c, d)$ during a finite interval of time $\left[0, \tau_{2}\right)$ with $c \in C\left(\left[0, \tau_{2}\right), H^{3}\left(\mathbf{R}^{2}\right)\right)$ and $d \in C\left(\left[0, \tau_{2}\right), W^{2, \infty}\left(\mathbf{R}^{2}\right)\right)$.

\subsection{Multiple scales and pseudo-differential operators}

We first define the analytic framework in which we will work. Consider a function $u\left(x^{\prime}, z^{\prime}\right)$, with $z^{\prime} \in \mathbf{R}^{n}$ and $x^{\prime} \in \mathbf{T}^{n}=\mathbf{R}^{n} / \Gamma$, where $\Gamma$ is the lattice with respect to which $u$ is periodic in $x^{\prime}$. For $x^{\prime} \in \mathbf{R}^{n}$ and $0<\epsilon$, we define a multiple scale function as a function $u\left(x^{\prime}, z^{\prime}\right)$, evaluated on the subspace $\left\{z^{\prime}=\epsilon x^{\prime}\right\} \subseteq \mathbf{R}^{2 n}$. We will use the notation $u\left(x^{\prime}, \epsilon x^{\prime}\right)$ or $\left.u\left(x^{\prime}, z^{\prime}\right)\right|_{z^{\prime}=\epsilon x^{\prime}}$ for such functions. For functions $u=u\left(x^{\prime}\right)$ (which may depend upon $\epsilon$ as well), the usual Sobolev $W^{s, q}$-norm is written $\|u\|_{s, q}$. When a multiple scale function $c$ depends upon the slow variables alone, that is, $c=c\left(\epsilon x^{\prime}\right)$, it is measured in a Sobolev norm in that variable, $|c|_{s, q}=\sum_{|\alpha| \leq s}\left(\int\left|\partial_{z^{\prime}}^{\alpha} c\right|^{q} d z^{\prime}\right)^{1 / q}$. Scaling by $\epsilon$ provides a relationship between these two norms; $\|c\|_{s, q} \leq \epsilon^{-n / q}|c|_{s, q}$ for any $\epsilon \leq 1$. For the expected modulational form of approximate solutions to the water wave problem, a third norm is used for estimating multiple scale functions. For 
$u=u\left(x^{\prime}, \epsilon x^{\prime}\right)$, we define

$$
|u|_{(a, b), q}=\sum_{\substack{|\alpha| \leq a \\|,| \leq \mid \leq b}}\left(\int_{\mathbf{R}^{n}} \int_{\mathbf{T}^{n}}\left|\partial_{x^{\prime}}^{\alpha} \partial_{z^{\prime}}^{\beta} u\left(x^{\prime}, z^{\prime}\right)\right|^{q} d x^{\prime} d z^{\prime}\right)^{1 / q} .
$$

The analysis of the modulational regime of the water wave problem involves classes of functions of the form $\left.u\left(x^{\prime}, z^{\prime}\right)\right|_{z^{\prime}=\epsilon x^{\prime}}=\left.\exp \left(i k x^{\prime}\right) c\left(z^{\prime}\right)\right|_{z^{\prime}=\epsilon x^{\prime}}$, for $z^{\prime} \in \mathbf{R}^{2}$, and the above discussion implies that

$$
\left|u\left(x^{\prime}, z^{\prime}\right)\right|_{(a, b), q} \leq C(k)\left|c\left(z^{\prime}\right)\right|_{b, q} .
$$

A norm related to (6.9) is used in [8] for the analysis of pseudo-differential operators and their actions on multiple scale functions. Here we give an analogous result on the bounds for pseudo-differential operators, using the $W^{s, q}$ norms as our reference point.

A Fourier multiplier operator is defined as

$$
M(D) u\left(x^{\prime}\right)=\frac{1}{(2 \pi)^{n}} \int e^{i k \cdot x^{\prime}} M(k) \hat{u}(k) d k .
$$

where $\hat{u}$ is the Fourier transform $\mathrm{m}$ of $u$. For $u$ a multiple scale function of $x^{\prime} \in \mathbf{T}^{n}, z^{\prime} \in \mathbf{R}^{n}, M(D) u$ depends upon $\epsilon$ and $x^{\prime}$, but is not in general a multiple scale function. However it does have an asymptotic expansion in terms of them.

THeOREM 6.3. - Assume that the Fourier multiplier $M(D)$ has the property that $\left|\partial_{k}^{j} M(k)\right| \leq c_{j}\left(1+|k|^{2}\right)^{(m-j) / 2}, 0 \leq j \leq m$. Then its operation on a multiple scale function has an asymptotic expansion

$$
\begin{aligned}
(M(D) u)\left(x^{\prime} ; \epsilon\right)= & \left(\sum_{j=0}^{N} \frac{\epsilon^{j}}{j !} \partial_{k}^{j} M\left(D_{x^{\prime}}\right)\left(\frac{1}{i} \partial_{z^{\prime}}\right)^{j} u\right)\left(x^{\prime}, \epsilon x^{\prime}\right) \\
& +R_{N+1} u .
\end{aligned}
$$

The remainder has the estimate

$$
\left\|R_{N \mid 1} u\right\|_{s, q} \leq C_{s q} \epsilon^{N+1}|u|_{\left(\ell_{1}, \ell_{2}\right), q}
$$

where the subscripts are $\ell_{1}=m-(N+1)+s+\sigma, \ell_{2}=s+\sigma+$ $\max (m, N+1)$ for any $\sigma>n$.

Given the analysis of reference [8], the proof of this result is relatively standard. For sake of completeness however, we give it in Appendix B. 


\subsection{Estimates on the water-wave operator acting on the modulational approximation}

As noted before, the character of the solutions of the Davey-Stewartson system is different whether the cquation for $d$ is of clliptic $(m>0)$ or hyperbolic type $(m<0)$. Thus, we will divide the analysis into these two cases referred to as case I and case II respectively.

In case I, starting with an initial condition $c_{0} \in H^{r}\left(\mathbf{R}^{2}\right)$, there exists a unique solution $(c, d)$, with $c$ and $d$ being continuous functions of $\tau \in\left[0, \tau_{1}\right]$ with values in $H^{r}\left(\mathbf{R}^{2}\right)$ and $H^{r+1}\left(\mathbf{R}^{2}\right)\left(\tau_{1}\right.$ may be finite or infinite) and by Sobolev embedding in $W^{s, q}$ and $W^{s-1, q}$ for $s=r-\frac{1}{2}\left(\frac{1}{2}-\frac{1}{q}\right)$. From this solution, we construct a functional of the modulation approximation $\widetilde{\eta}=\widetilde{\eta}(c, d)$ and $\widetilde{\xi}=\widetilde{\xi}(c, d)$ using the expressions (6.4-7) in the time interval $I=\left[0, \epsilon^{-2} \tau_{1}\right]$. We will establish an upper bound for $\mathbf{W}(\widetilde{\eta}, \widetilde{\xi})$ in the interval $I$ in a Sobolev norm in terms of powers of $\epsilon$ and Sobolev norms of $c$ and $d$.

In case II, we start again with an initial condition in $H^{r}\left(\mathbf{R}^{2}\right)$. There exists a unique solution $(c, d)$ with $c$ being continuous function of $\tau$ in $\left[0, \tau_{2}\right]$ with values in $H^{r}\left(\mathbf{R}^{2}\right)$ and thus in $W^{s, q}\left(\mathbf{R}^{2}\right)$ and $d$ a continuous function of $\tau$ with values only in $W^{s-1, \infty}$ and thus not integrable. To overcome this difficulty, we introduce a cut-off function $\chi$ infinitely differentiable with compact support and we construct $\bar{\eta}=\widetilde{\eta}(c, \chi d)$ and $\bar{\xi}=\widetilde{\xi}(c, \chi d)$. An upper bound will be established for $\mathbf{W}(\bar{\eta}, \bar{\xi})$.

In both cases, an important point is the amount of derivative loss. To quantify these amounts, we introduce the notion of estimating factors which are functions $F\left(s_{1}, s_{2}\right)$ that are non-negative, continuous, and satisfy $F(0,0)=0$, with polynomial growth in $\left(s_{1}, s_{2}\right)$, (and depending on $k$ : and $\omega)$.

LEMMA 6.4. - Set $\tilde{\eta}$ and $\tilde{\xi}$ as in (6.4-7), the approximations to the operator $G(\eta)$ of Section 4 satisfy

$$
\begin{aligned}
& \left\|\left(G(\widetilde{\eta})-G_{0}\right) \tilde{\xi}\right\|_{s, q} \\
& \quad \leq \epsilon^{2} F\left(\sup _{t \in I}|c|_{C, 1,3}, \sup _{t \in I}|d|_{C^{* 12}}\right)\left(\|c\|_{s+2, q}+\|d\|_{s+1, q}\right) \\
& \left\|\left(G(\widetilde{\eta})-G_{0}-G_{1}(\widetilde{\eta})\right) \widetilde{\xi}\right\|_{s, q} \\
& \quad \leq \epsilon^{3} F\left(\sup _{t \in I}|c|_{C^{s+3},} \sup _{t \in I}|d|_{C^{s+2}}\right)\left(\|c\|_{s+2, q}+\|d\|_{s+1, q}\right) \\
& \left\|\left(G(\widetilde{\eta})-G_{0}-G_{1}(\widetilde{\eta})-G_{2}(\widetilde{\eta})\right) \widetilde{\xi}\right\|_{s, q} \\
& \quad \leq \epsilon^{4} F\left(\sup _{t \in I}|c|_{C^{s+3}}, \sup _{t \in I}|d|_{C^{s+2}}\right)\left(\|c\|_{s+2, q}+\|d\|_{s+1, q}\right)
\end{aligned}
$$


Proof. - An application of Theorem 4.7 gives:

$$
\left\|R_{j}(\widetilde{\eta}) \tilde{\xi}\right\|_{s, q} \leq C|\widetilde{\eta}|_{C^{1}}^{j-1}\left(|\widetilde{\eta}|_{C^{1}}\|\tilde{\xi}\|_{s+1, q}+C(s)|\widetilde{\eta}|_{C^{s+1}}\|\tilde{\xi}\|_{1, q}\right)
$$

for $j=1,2,3$. We have furthermore from the approximate solutions and Gagliardo-Nirenberg inequalities:

$$
\begin{gathered}
\|\tilde{\xi}\|_{s+1, q} \leq \epsilon F_{1}\left(\sup _{t \in I}|c|_{C^{0}}\right)\left(\|c\|_{s+2, q}+\|d\|_{s+1, q}\right) \\
\|\widetilde{\eta}\|_{C^{s+1}} \leq \epsilon F_{2}\left(\sup _{t \in I}|c|_{C^{s+3}}, \sup _{t \in I}|d|_{C^{s+2}}\right) .
\end{gathered}
$$

THEOREM 6.5. - Consider the case $m>0$. Let $(c, d)$ be a solution of the Davey-Stewartson system (3.24). The approximate solution $(\widetilde{\eta}, \widetilde{\xi})(c, d)$ satisfies the estimate:

$$
\begin{aligned}
& \sup _{t \in I}\|\mathbf{W}(\tilde{\eta}, \widetilde{\xi})\|_{s, q} \\
& \quad \leq \epsilon^{4-2 / q} F\left(\sup _{t \in I}|c|_{C^{s+3}}, \sup _{t \in I}|d|_{C^{s+2}}\right)\left(|c|_{s+6, q}+|d|_{s+6, q}\right)
\end{aligned}
$$

Proof. - We start with

$$
\begin{aligned}
W_{1}(\widetilde{\eta}, \widetilde{\xi}) & =\partial_{t} \tilde{\eta}-G(\widetilde{\eta}) \tilde{\xi} \\
& =\partial_{t} \tilde{\eta}-\left(G_{0}+G_{1}(\tilde{\eta})+G_{2}(\tilde{\eta})\right) \tilde{\xi}+R_{3}(\widetilde{\eta}) \tilde{\xi}
\end{aligned}
$$

We have shown that the last term can be estimated by

$$
\begin{aligned}
& \left\|R_{3}(\tilde{\eta}) \widetilde{\xi}\right\|_{s, q} \\
& \quad \leq \epsilon^{4} F\left(\sup _{t \in I}|c|_{C^{s+3}}, \sup _{t \in I}|d|_{C^{s+2}}\right)\left(\|c\|_{s+2, q}+\|d\|_{s+1, q}\right) \\
& \quad \leq \epsilon^{4-2 / q} F\left(\sup _{t \in I}|c|_{C^{s+3}}, \sup _{t \in I}|d|_{C^{s+2}}\right)\left(|c|_{s+2, q}+|d|_{s+1, q}\right)
\end{aligned}
$$

Since we consider the expression as a multiple scale function, we replace $\partial_{t}$ by $\partial_{t}-\epsilon \omega^{\prime} \partial_{z_{1}}+\epsilon^{2} \partial_{\tau}$. The computation of $\partial_{t} \tilde{\eta}$ is similar to the one performed in [8, eq.(5.7)]. We obtain an expression at order $\epsilon^{3}$ with a remainder of the form

$$
R_{4}=\epsilon^{4}\left(-\omega^{\prime} \partial_{z_{1}} \eta^{(3)}+\partial_{\tau} \eta^{(2)}\right)+\epsilon^{5} \partial_{\tau} \eta^{(3)}
$$

Using the expressions of $\eta^{(2)}$ and $\eta^{(3)}$ in terms of $c$ and $d$ given in (6.6) and (6.7) and the fact that, when $m>0$, we have the estimate [13]

$$
\left\|\partial_{z_{\perp}} d\right\|_{q} \leq\left. C\|\| c\right|^{2} \|_{q}
$$

Vol. $14, n^{\circ} 5-1997$ 
the $W^{s, q}$ norm of $R_{4}$ is bounded by

$$
\epsilon^{4} F\left(\sup _{t \in I}|c|_{C^{s+2}}, \sup _{t \in I}|d|_{C^{s+1}}\right)\left(\|c\|_{s+4, q}+\|d\|_{s+2, q}\right)
$$

Next, we use the multiple scale approximations for the integral operators $\left(G_{0}+G_{1}(\widetilde{\eta})+G_{2}(\widetilde{\eta})\right) \widetilde{\xi}$. When applied to $\xi^{(1)}$, we expand $G_{0}$ up to order $\epsilon^{2}$ :

$$
\begin{aligned}
G_{0} \xi^{(1)}= & \epsilon k_{1} \sigma c e^{i \varphi}-i \epsilon^{2}\left(\sigma+h k_{1}\left(1-\sigma^{2}\right)\right) c_{z_{1}} e^{i \varphi} \\
& -\epsilon^{3}\left(\left(\frac{\sigma}{2 k_{1}}+\frac{h}{2}\left(1-\sigma^{2}\right)\right) c_{z_{2} z_{2}}\right. \\
& \left.+\left(h\left(1-\sigma^{2}\right)-h^{2} k_{1} \sigma\left(1-\sigma^{2}\right)\right) c_{z_{1} z_{1}}\right) e^{i \varphi}+c \cdot c \\
& -\epsilon^{3} h\left(d_{z_{1} z_{1}}+d_{z_{2} z_{2}}\right)+R_{4}
\end{aligned}
$$

A direct application of Theorem 6.3 gives

$$
\left\|R_{4}\right\|_{s, q} \leq C \epsilon^{4}\left(\|c\|_{s+6, q}+\|d\|_{s+6, q}\right)
$$

When applied to $\epsilon^{2} \xi^{(2)}$ (resp. $\epsilon^{3} \xi^{(3)}$ ), we expand $G_{0}$ to order $\epsilon$ (resp. zeroth order). We next address $G_{1}(\widetilde{\eta}) \widetilde{\xi}$. We recall that

$$
G_{1}(\widetilde{\eta}) \widetilde{\xi}=D \cdot \widetilde{\eta} D \widetilde{\xi}-G_{0} \widetilde{\eta} G_{0} \tilde{\xi}
$$

To expand $G_{1}(\tilde{\eta}) \tilde{\xi}$, we first write

$$
D \cdot \widetilde{\eta} D \widetilde{\xi}=\left(D_{1}^{(0)}+\epsilon D_{1}^{(1)}\right) \widetilde{\eta}\left(D_{1}^{(0)}+\epsilon D_{1}^{(1)}\right) \widetilde{\xi}+\epsilon^{2} D_{2}^{(1)} \widetilde{\eta} D_{2}^{(1)} \widetilde{\xi}
$$

Substituting $\widetilde{\eta}$ and $\widetilde{\xi}$ by their expressions (6.4), D. $\widetilde{\eta} D \widetilde{\xi}$ has an expansion in $\epsilon$ up to order three and a remainder $R_{4}$ bounded in $W^{s, q}$-norm by:

$$
c^{4} F\left(\sup _{t \in I}|c|_{C^{3}}, \sup _{t \in I}|d|_{C^{2}}\right)\left(\|c\|_{s+3, q}+\|d\|_{s+2, q}\right) .
$$

For the second term of $G_{1}$, we use Theorem 4.2 of [8] on composition of operators and get:

$$
\begin{aligned}
G_{0} \widetilde{\eta} G_{0} \tilde{\xi} & =-\epsilon^{2} G^{(0)} \eta^{(1)} G^{(0)} \xi^{(1)}-\epsilon^{3}\left(G^{(0)} \eta^{(2)} G^{(0)} \xi^{(1)}\right. \\
& +G^{(0)} \eta^{(1)} G^{(0)} \xi^{(2)}+G^{(0)} \eta^{(1)}\left(\tanh \left(h D_{1}^{(0)}\right) D_{1}^{(1)}\right. \\
& \left.+h D_{1}^{(0)}\left(1-\tanh ^{2}\left(h D_{1}^{(0)}\right)\right) D_{1}^{(1)}\right) \xi^{(1)}+\left(\tanh \left(h D_{1}^{(0)}\right) D_{1}^{(1)}\right. \\
& \left.\left.+h D_{1}^{(0)}\left(1-\tanh ^{2}\left(h D_{1}^{(0)}\right)\right) D_{1}^{(1)}\right) \eta^{(1)} G^{(0)} \xi^{(1)}\right)+R_{4}
\end{aligned}
$$


Again, the remainder $R_{4}$ is bounded in $W^{s, q}$-norm by

$$
\epsilon^{4} F\left(\sup _{t \in I}|c|_{C^{s+3}}, \sup _{t \in I}|d|_{C^{s+2}}\right)\left(\|c\|_{s+5, q}+\|d\|_{s+5, q}\right)
$$

A similar computation is done for $G_{2}(\widetilde{\eta}) \widetilde{\xi}$. Combining all these results, it follows that, by the construction of Section 3 , in the expansion of $W_{1}(\widetilde{\eta}, \widetilde{\xi})$, the terms up to order $\epsilon^{3}$ cancel and the remainder has a $W^{s, q}$-norm bounded from above by the r.h.s of (6.19).

The same computation (although a little heavier) is performed for $W_{2}(\widetilde{\eta}, \widetilde{\xi})$. This completes the proof of Theorem 6.5 devoted to the case $m>0$. We turn now to the case $m<0$.

As mentioned before, the function $d$ is in $W^{s, \infty}\left(\mathbf{R}^{2}\right)$ without being in any $q$-integrable spaces. This is due to the hyperbolic nature of the second equation of the Davey-Stewartson system. In this case, we have a local result. Let $\chi\left(z^{\prime}\right) \subset C_{0}^{\infty}\left(R^{2}\right)$ a cutoff function such that $\chi\left(z^{\prime}\right)=1$ for $\left|z^{\prime}\right|<R$. Consider the approximate solutions $\bar{\eta}=\widetilde{\eta}(c, \chi d)$ and $\vec{\xi}=\widetilde{\xi}(c, \chi d)$, where $(c, d)$ satisfies the Davey-Stewartson system.

THEOREM 6.6. - Let $(c, d)$ solve the Davey-Stewartson system in the case $m<0$. The truncated approximate solution $(\bar{\eta} \bar{\xi})$ satisfies the estimate:

$$
\begin{aligned}
& \sup _{t \in I}\left\|\chi_{1}\left(z^{\prime}\right) \mathbf{W}(\bar{\eta}, \bar{\xi})\right\|_{s, q} \\
& \quad \leq \epsilon^{4-2 / q} F\left(\sup _{t \in I}|c|_{C^{s+3}}, \sup _{t \in I}|d|_{C^{s+2}}\right)\left(|c|_{s+6, q}+|\chi d|_{s+6, q}\right)
\end{aligned}
$$

for all $\chi_{1} \in C_{0}^{\infty}\left(R^{2}\right)$ with $\operatorname{supp}\left(\chi_{1}\right) \subseteq B_{R}(0)$

Proof. - It follows the steps of the proof of Theorem 6.5: Again, we separate $G(\bar{\eta})$ in the form

$$
G(\bar{\eta})=\left(G_{0}+G_{1}(\bar{\eta})+G_{2}(\bar{\eta})\right)+R_{3}(\bar{\eta})
$$

and apply Lemma 6.4 to estimate the remainder. When computing $\mathbf{W}(\bar{\eta}, \bar{\xi})$, we use the multiple scale approximations and get that $\mathbf{W}(\bar{\eta}, \bar{\xi})$ is equal to a functional of the Davey-Stewartson operator applied to $(c, \chi d)$ plus a remainder of the order of $O\left(\epsilon^{4}\right)$. When the support of $\chi_{1}$ is included in a set on which $\chi d=d, \chi_{1} \mathbf{W}(\bar{\eta}, \bar{\xi})$ reduces to a remainder of order $O\left(\epsilon^{4}\right)$. A localized version of the same conclusion then follows. 


\section{ACKNOWLEDGEMENTS}

The work of the first author was partially supported by the National Science Fundation grant DMS-9401377 during the time that this research was performed, and the work of the third author by NSERC operating grant OGPIN16.

\section{APPENDIX A}

This appendix is devoted to the computation of the Fourier transforms of various kernels arising in the derivation of the Taylor expansion of $G$. Let $\mathcal{F}$ denote the Fourier transform. In the following, $\mathbf{k} \in \mathbf{R}^{2}$ will be the variable in the Fourier space and $\mathbf{x} \in \mathbf{R}^{2}$ the variable in the physical space.

Proposition A.1

$$
\mathcal{F}\left(\frac{1}{\left(|x|^{2}+4 h^{2}\right)^{1 / 2}}\right)=2 \pi \frac{1}{|k|} e^{-2 h|k|}
$$

Corollary A.1

$$
\begin{aligned}
& \mathcal{F}\left(\frac{1}{|x|}\right)=2 \pi \frac{1}{|k|} \\
& \mathcal{F}\left(\frac{x_{j}}{\left(|x|^{2}+4 h^{2}\right)^{3 / 2}}\right)=-2 i \pi \frac{k_{j}}{|k|} e^{-2 h|k|} \quad(j=1,2) \\
& \mathcal{F}\left(v p \frac{x_{j}}{|x|^{3}}\right)=-2 i \pi \frac{k_{j}}{|k|}
\end{aligned}
$$

Corollary A.2

$$
\begin{aligned}
& \mathcal{F}\left(\frac{1}{|x|^{3}}\right)=-2 \pi|k| \\
& \mathcal{F}\left(\frac{2 h}{\left(|x|^{2}+4 h^{2}\right)^{3 / 2}}\right)=2 \pi e^{-2 h|k|} \\
& \mathcal{F}\left(\frac{24 h^{3}}{\left(|x|^{2}+4 h^{2}\right)^{5 / 2}}\right)=2 \pi(1+2 h|k|) e^{-2 h|k|}
\end{aligned}
$$

COROllary A.3

$$
\begin{aligned}
& \mathcal{F}\left(\frac{2 h x_{j}}{\left(|x|^{2}+4 h^{2}\right)^{5 / 2}}\right)=-\frac{2 \pi}{3} i k_{j} e^{-2 h|k|} \\
& \mathcal{F}\left(\frac{16 h^{5}}{\left(|x|^{2}+4 h^{2}\right)^{7 / 2}}\right)=2 \pi\left(\frac{1}{10}(1+2 h|k|)+\frac{2}{15} h^{2}|k|^{2}\right) e^{-2 h|k|}
\end{aligned}
$$


Let us first obtain the Corollaries from proposition A.1:

Proof of Corollary A.1. - Equality (A.2) is obtained from (A.1) by taking the limit $h \rightarrow 0$. Equality (A.3) is obtained from (A.1) by differentiation and (A.4) as the limit of (A.3) when $h \rightarrow 0$.

Proof of Corollary A.2. - To get (A.5), we notice that $\frac{1}{|x|^{3}}=-\Delta\left(\frac{1}{|x|}\right)$ and to get (A.6), we rewrite (A.1) as

$$
\frac{\partial}{\partial k_{j}} \mathcal{F}\left(\frac{1}{\left(|x|^{2}+4 h^{2}\right)^{3 / 2}}\right)=\frac{1}{2 h} \frac{\partial}{\partial k_{j}} e^{-2 h|k|} .
$$

Finally, (A.7) is obtained from the identity

$$
\Delta\left(\frac{1}{\left(|x|^{2}+4 h^{2}\right)^{1 / 2}}\right)=\frac{1}{\left(|x|^{2}+4 h^{2}\right)^{3 / 2}}-\frac{12 h^{2}}{\left(|x|^{2}+4 h^{2}\right)^{5 / 2}} .
$$

and (A.1) and (A.6).

Proof of Corollary A.3. - Equality (A.8) is a direct consequence of (A.6) and (A.9) results from the computation of $\Delta\left(\frac{1}{\left(|x|^{2}+4 h^{2}\right)^{3 / 2}}\right)$.

Proof of Proposition A.1. - Let us compute $\mathcal{F}^{-1}\left(\frac{1}{|k|} e^{-2 h|k|}\right)$ :

$$
\begin{aligned}
\mathcal{F}^{-1}\left(\frac{1}{|k|} e^{-2 h|k|}\right) & =\frac{1}{(2 \pi)^{2}} \int \frac{1}{|k|} e^{i k \cdot x} e^{-2 h|k|} d k \\
& =\frac{1}{(2 \pi)^{2}} \int_{0}^{2 \pi} \int_{0}^{\infty} e^{-2 h \alpha} e^{i|x| \alpha \cos \varphi} d \alpha d \varphi \\
& =\frac{1}{(2 \pi)^{2}} \int_{0}^{2 \pi} \frac{1}{2 h-i|x| \cos \varphi} d \varphi
\end{aligned}
$$

After some elementary computations, we get

$$
\mathcal{F}^{-1}\left(\frac{1}{|k|} e^{-2 h|k|}\right)=\frac{h}{\pi^{2}} \int_{0}^{\pi} \frac{1}{|x|^{2} \cos ^{2} \varphi+4 h^{2}} d \varphi
$$

Using the change of variable $\theta=\tan \varphi$, we get

$$
\mathcal{F}^{-1}\left(\frac{1}{|k|} e^{-2 h|k|}\right)=\frac{j}{2 \pi} \frac{1}{\left(|x|^{2}+4 h^{2}\right)^{1 / 2}}
$$

Vol. $14, n^{\circ}$ 5-1997. 


\section{APPENDIX B}

This appendix is devoted to the proof of Theorem 6.3. The Fourier multiplier operator $M(D)$ is defined as

$$
M(D) u(x)=\frac{1}{(2 \pi)^{n}} \int e^{i \xi \cdot x} m(\xi) \hat{u}(\xi) d \xi .
$$

and has the property that $\left|\partial_{\xi}^{\alpha} m(\xi)\right| \leq c_{j}\left(1+|\xi|^{2}\right)^{(m-|\alpha|) / 2}, 0 \leq \alpha \leq m$. Let $u(x, \epsilon x)=\left.u(x, z)\right|_{z=\epsilon x}$ be a multiple scale function with $x \in \mathbf{T}^{n}$ and $z \in \mathbf{R}^{n}$. When applied to $u(x, \epsilon x)$ Fourier multipliers have the asymptotic expansion in multiscale functions

$$
(M(D) u)(x ; \epsilon)=\left(\sum_{j=0}^{N} \frac{\epsilon^{j}}{j !} \partial_{\xi}^{j} M\left(D_{x}\right)\left(-i \partial_{z}\right)^{j} u\right)(x, \epsilon x)+R_{N+1} u
$$

(see eq (4.8) of ref. [8] for details), with the remainder term $R_{N+1} u$ given by

$$
\begin{aligned}
& R_{N+1} u(x ; \epsilon)=\epsilon^{N+1} \int e^{i(\xi+\epsilon \eta) x} e^{-i\left(\xi x^{\prime}+\eta z^{\prime}\right)} \\
& \quad\left(\int_{0}^{1} \frac{t^{N}}{N !} \partial_{\xi}^{N+1} m(\xi+\epsilon t \eta) d t\right) D_{z}^{N+1} u\left(x^{\prime}, z^{\prime}\right) d x^{\prime} d z^{\prime} d \xi d \eta
\end{aligned}
$$

The order of $\partial_{\xi}^{N+1} m(\cdot)$ is $m-(N+1)$. To estimate $R_{N+1} u$, we separate the domain of spatial integration in four parts:

(i) $\left|x-x^{\prime}\right|>1 / 2$ and $\left|\epsilon x-z^{\prime}\right|>1 / 2$,

(ii) $\left|x-x^{\prime}\right| \leq 3 / 2$ and $\left|\epsilon x-z^{\prime}\right| \leq 3 / 2$,

(iii) $\left|x-x^{\prime}\right|>1 / 2$ and $\left|\epsilon x-z^{\prime}\right| \leq 3 / 2$,

(iv) $\left|x-x^{\prime}\right| \leq 3 / 2$ and $\left|\epsilon x-z^{\prime}\right|>1 / 2$,

focussing on the individual regions using a smooth partition of unity $\left\{\chi^{(j)}\left(x, x^{\prime}, z^{\prime}\right)\right\}$ adapted to this decomposition. We denote by $R_{N+1}^{(i)} u$, $R_{N+1}^{(i i)} u \ldots$ their corresponding contribution to $R_{N+1} u$.

Over the first region, the contribution to the error term is

$$
\begin{aligned}
R_{N+1}^{(i)} u= & \epsilon^{N+1} \int e^{i(\xi+\epsilon \eta) x} e^{-i\left(\xi x^{\prime}+\eta z^{\prime}\right)}\left(\frac{1}{i\left(x-x^{\prime}\right)} \frac{1}{i\left(\epsilon x-z^{\prime}\right)}\right)^{\gamma} \\
& \times\left(\frac{1}{i} \partial_{\xi}\right)^{\gamma}\left(\frac{1}{i} \partial_{\eta}\right)^{\gamma}\left(\int_{0}^{1} \frac{t^{N}}{N !} \partial_{\xi}^{N+1} m(\xi+\epsilon t \eta) d t\right) \\
& \times D_{z}^{N+1} u\left(x^{\prime}, z^{\prime}\right) \chi^{(j)}\left(x, x^{\prime}, z^{\prime}\right) d x^{\prime} d z^{\prime} d \xi d \eta
\end{aligned}
$$


We estimate the oscillatory integrand to be

$$
\left|\partial_{\xi}^{\gamma} \partial_{\eta}^{\gamma} \partial_{\xi}^{N+1} m(\xi+\epsilon t \eta)\right| \leq C_{\gamma}(\epsilon t)^{\gamma}\left(1+|\xi+\epsilon t \eta|^{2}\right)^{m-(N+1)-2 \gamma} .
$$

Now, we separate the contributions $|\eta|<3|\xi| / 2$ and $|\eta|>|\xi| / 2$, with similar smooth cutoff functions $\hat{\chi}^{(1)}(\xi, \eta), \hat{\chi}^{(2)}(\xi, \eta)$. When $|\eta|<3|\xi| / 2$, the oscillatory integral is absolutely convergent as soon as $\gamma>(m-(N+$ 1)) $/ 2+n / 2$. Furthermore, the integrand

$$
\begin{aligned}
& \int e^{i(\xi+\epsilon \eta) x} e^{-i\left(\xi x^{\prime}+\eta z^{\prime}\right)}\left(\frac{1}{i\left(x-x^{\prime}\right)} \frac{1}{i\left(\epsilon x-z^{\prime}\right)}\right)^{\gamma} \\
& \times\left(\frac{1}{i} \partial_{\xi}\right)^{\gamma}\left(\frac{1}{i} \partial_{\eta}\right)^{\gamma}\left(\int_{0}^{1} \frac{t^{N}}{N !} \partial_{\xi}^{N+1} m(\xi+\epsilon t \eta) d t \hat{\chi}^{(1)}(\xi, \eta)\right) d \xi d \eta
\end{aligned}
$$

is integrable in $\left(x^{\prime}, z^{\prime}\right)$, uniformly in $x$, and is also integrable in $x$, uniformly over $\left(x^{\prime}, z^{\prime}\right)$. Note that no additional derivatives act on the function $u$.

When $|\eta|>|\xi| / 2$, we rewrite the contribution in the form

$$
\begin{aligned}
& \epsilon^{N+1} \int e^{i(\xi+\epsilon \eta) x} e^{-i\left(\xi x^{\prime}+\eta z^{\prime}\right)}\left(\frac{1}{i\left(x-x^{\prime}\right)} \frac{1}{i\left(\epsilon x-z^{\prime}\right)}\right)^{\gamma} \\
& \quad\left(\int_{0}^{1}(\epsilon t)^{\gamma} \frac{t^{N}}{N !}\left(\frac{1}{i} \partial_{\xi}\right)^{\gamma}\left(\frac{1}{i} \partial_{\eta}\right)^{\gamma} \partial_{\xi}^{N+1} m(\xi+\epsilon t \eta) d t \hat{\chi}^{(2)}(\xi, \eta)\right) \\
& \quad \times\left(1+|\xi|^{2}\right)^{-\delta_{1} / 2}\left(1+|\eta|^{2}\right)^{-\delta_{2} / 2} \\
& \quad \times\left(1-\Delta_{x^{\prime}}\right)^{\delta_{1} / 2}\left(1-\Delta_{z^{\prime}}\right)^{\delta_{2} / 2} D_{z}^{N+1} u\left(x^{\prime}, z^{\prime}\right) d x^{\prime} d z^{\prime} d \xi d \eta
\end{aligned}
$$

To have an absolute convergent integral, we choose $\delta_{1}>n$ and $\delta_{2}>n$. This places $\delta_{1}$ derivatives with respect to $x^{\prime}$ and $N+1+\delta_{2}$ derivalives with respect to $z^{\prime}$ on $u$.

Now we turn to $R_{N+1}^{(i i)} u$, paying less heed than we should to the smoothness of $u$. We rewrite it in a form where all derivatives act on $u\left(x^{\prime}, z^{\prime}\right)$ :

$$
\begin{aligned}
R_{N+1}^{(i i)} u= & \epsilon^{N+1} \int e^{i(\xi+\epsilon \eta) x} e^{-i\left(\xi x^{\prime}+\eta z^{\prime}\right)}\left(1+|\xi|^{2}\right)^{-\gamma_{1} / 2}\left(1+|\eta|^{2}\right)^{-\gamma_{2} / 2} \\
& \times\left(\int_{0}^{1} \frac{t^{N}}{N !} \partial_{\xi}^{N+1} m(\xi+\epsilon t \eta) d t\right) \\
& \left(1-\Delta_{x^{\prime}}\right)^{\delta_{1} / 2}\left(1-\Delta_{z^{\prime}}\right)^{\gamma_{2} / 2} D_{z}^{N+1} u\left(x^{\prime}, z^{\prime}\right) d x^{\prime} d z^{\prime} d \xi d \eta . \quad \text { (B.6) }
\end{aligned}
$$

Notice that the spatial domain is uniformly finite in $x$ for each $\left(x^{\prime}, z^{\prime}\right)$. Choosing $\gamma_{1}, \gamma_{2}>n+m-(N+1)$, the oscillatory integrals are absolutely convergent. In this term, we have at most $n+m-(N+1)$ derivatives Vol. $14, n^{\circ}$ 5-1997. 
with respect to $x^{\prime}$ and $n+m-(N+1)+(N+1)$ derivatives with respect to $z^{\prime}$ on $u$.

We rewrite $R_{N+1}^{(i i i)} u$ as

$$
\begin{aligned}
R_{N+1}^{(i i i)} u= & \left.\epsilon^{N+1} \int e^{i(\xi+\epsilon \eta) x} e^{-i\left(\xi x^{\prime}+\eta z^{\prime}\right)}\left(\frac{1}{i\left(x-x^{\prime}\right)}\right)\right)^{\gamma} \\
& \times\left(\int_{0}^{1} \frac{t^{N}}{N !}\left(\frac{1}{i} \partial_{\xi}\right)^{\gamma} \partial_{\xi}^{N+1} m(\xi+\epsilon t \eta) d t\right) \\
& \times D_{z}^{N+1} u\left(x^{\prime}, z^{\prime}\right) d x^{\prime} d z^{\prime} d \xi d \eta
\end{aligned}
$$

Here we separate the contributions where $|\eta|<3|\xi| / 2$ and $|\eta|>|\xi| / 2$ and proceed as in case (i). We thus choose $\gamma>n$ and $\gamma>m-(N+1)$. The count of derivatives gives at least $n+m-(N+1)$ derivatives with respect to $x^{\prime}$ and $n+(N+1)$ derivatives with respect to $z^{\prime}$ on $u$.

The last term to estimate is $R_{N+1}^{(i v)} u$.

$$
\begin{aligned}
R_{N+1}^{(i \mathrm{v})} u= & \epsilon^{N+1} \int e^{i(\xi+\epsilon \eta) x} e^{-i\left(\xi x^{\prime}+\eta z^{\prime}\right)}\left(\frac{1}{i\left(\epsilon x-z^{\prime}\right)}\right)^{\gamma} \\
& \times\left(\int_{0}^{1} \frac{t^{N}}{N !}(\epsilon t)^{\gamma}\left(\frac{1}{i} \partial_{\eta}\right)^{\gamma} \partial_{\xi}^{N+1} m(\xi+\epsilon t \eta) d t\right) \\
& \times D_{z}^{N+1} u\left(x^{\prime}, z^{\prime}\right) d x^{\prime} d z^{\prime} d \xi d \eta
\end{aligned}
$$

Take $\gamma>n$ and $\gamma>m-(N+1)$. Then, $\partial_{\eta}^{\gamma} \partial_{\xi}^{N+1} m$ will be bounded and the power $\gamma$ of $1 /\left|\epsilon x-z^{\prime}\right|^{\gamma}$ will be integrable in $z^{\prime}$, uniformly over $x$. Note that for given $x, x^{\prime}$ varies over a uniformly bounded cube. Furthermore, for fixed $\left(x^{\prime}, z^{\prime}\right)$, the $x$-integral also varies over a uniformly bounded cube. To bound the oscillatory integral, we separate the contributions where $|\eta|<3|\xi| / 2$ and $|\eta|>|\xi| / 2$, again by smooth cutoff functions. When $|\eta|<|\xi| / 2$, we choose $\gamma>n$. When $|\eta|>|\xi| / 2$, we follow the procedure above and write this contribution as:

$$
\begin{aligned}
\epsilon^{N+1} & \int e^{\imath(\xi+\epsilon \eta) x} e^{-i\left(\xi x^{\prime}+\eta z^{\prime}\right)}\left(\frac{1}{i\left(\epsilon x-z^{\prime}\right)}\right)^{\gamma} \\
& \times\left(\int_{0}^{1}(\epsilon t)^{\gamma} \frac{t^{N}}{N !}\left(\frac{1}{i} \partial_{\eta}\right)^{\gamma} \partial_{\xi}^{N+1} m(\xi+\epsilon t \eta) d t\right) \\
& \times\left(1+|\xi|^{2}\right)^{-\gamma_{1} / 2}\left(1+|\eta|^{2}\right)^{-\gamma_{2} / 2} \\
& \left(1-\Delta_{x^{\prime}}\right)^{\gamma / 2}\left(1-\Delta_{z^{\prime}}\right)^{\gamma_{2} / 2} D_{z}^{N+1} u\left(x^{\prime}, z^{\prime}\right) d x^{\prime} d z^{\prime} d \xi d \eta
\end{aligned}
$$

Here we choose $\gamma_{1}, \gamma_{2}>n$ and we have the same count as case (iii) above. This completes the proof of Theorem 6.3. 


\section{REFERENCES}

[1] M. J. Ablowitz and H. Segur, J. Fluid Mech., Vol. 92, 1979, pp. 691-715.

[2] D. J. Benney and G. J. Roskes, Stud. Appl. Math., Vol. 48, 1969, pp. 377-385.

[3] M. Christ and J. L. Journé, Acta Math., Vol. 159, 1987, pp. 51-80.

[4] R. R. Cotfman and Y. Meyer, Proc. Symp. Pure Math., Vol. 43, 1985, pp. 71-78.

[5] P. Collet and J. P. Eckmann, Comm. Math. Phys., Vol. 132, 1990, pp. 139-153.

[6] W. Craig, Comm. Part. Diff. Eq., Vol. 10, 1985, pp. 787-1003.

[7] W. Craig and M. Groves, Wave Motion, Vol. 19, 1994, pp. 367-389.

[8] W. Craig, C. Sulem and P. L. Sulem, Nonlinearity, Vol. 108, 1992, pp. 497-522.

[9] W. Craig and C. Sulem, J. Comp. Phys., Vol. 108, 1993, pp. 73-83.

[10] A. Davey and K. Stewartson, Proc. Roy. Soc. A, Vol, 338, 1974, pp. 101-110.

[11] V. D. DJoRdjEVIC and L. G. Redekopp, J. Fluid Mech., Vol. 79, 1977, pp. 703-714.

[12] P. R. Garabedian and M. Schiffer, J. Analyse Mathématique, Vol. 2, 1952, pp. 281-368.

[13] J. M. Ghidalia and J. C. Saut, Nonlinearity, Vol. 3, 1990, pp. 475-506.

[14] M. GuZMÁn-GómEZ, PhD thesis, University of Toronto, 1995.

[15] H. Hasimoto and H. ONo, J. Phys. Soc. Japan, Vol. 33, 1972, pp. 805-811.

[16] N. HAYASH and J. C. SaUt, Diff. and Integral Equations, Vol. 8, 1995, pp. 1657-1675.

[17] T. Kano and T. Nishida, J. Math Kyoto Univ., Vol. 19, 1979, pp. 335-370.

[18] T. Kano and T. Nishida, Osaka J. Math., Vol. 23, 1986, pp. 389-413.

[19] P. Kirrmann, G. Schneider and A. Mielke, Proceedings of the Royal Society of Edinburgh, Vol. 122A, 1992, pp. 85-91.

[20] F. Linares and G. PONCE, Ann. Inst. Poincaré, Analyse nonlinéaire, Vol. 10, 1993, pp. $523-548$

[21] R. de la Llave and P. Panayotaros, Water waves on the surface of the sphere, J. Nonlinear Science, Vol. 6, No. 2, 1996, pp. 147-167.

[22] A. Newell, Solitons in Mathematics and Physics, SIAM, Philadelphia, 1985.

[23] L. F. MCGoldrick, J. Fluid. Mech., Vol. 52, 1972, pp. 725-751.

[24] G. C. Papanicolaou, C. Sulem, P. L. Sulem and X. P. Wang, Physica D, Vol. 72, 1994 , pp. 61-86.

[25] R. PIERCE and E. WAYNE, On the validity of mean-field amplitude equations for counterpropagating wavetrains, Nonlinearity, Vol. 8, No. 5, 1995, pp. 769-779.

[26] M. Tsutsumi, J. Math. Anal. Appl., Vol. 82, 1994, pp. 680-704.

[27] V. E. Zakharov, J. Appl. Mech. Tech. Phys., Vol. 9, 1968, pp. 190-194.

(Manuscript received September 27, 1995; revised April 30, 1996.)

Vol. $14, \mathrm{n}^{\circ}$ 5-1997. 